\title{
Resonant Post-newtonian Eccentricity Excitation in Hierarchical Three-body Systems
}

\section{Citation}

Naoz, Smadar, Bence Kocsis, Abraham Loeb, and Nicolás Yunes. 2013. "RESONANT POSTNEWTONIAN ECCENTRICITY EXCITATION IN HIERARCHICAL THREE-BODY SYSTEMS." The Astrophysical Journal 773 (2): 187. https://doi.org/10.1088/0004-637x/773/2/187.

\section{Permanent link}

http://nrs.harvard.edu/urn-3:HUL.InstRepos:41393184

\section{Terms of Use}

This article was downloaded from Harvard University's DASH repository, and is made available under the terms and conditions applicable to Other Posted Material, as set forth at http:// nrs.harvard.edu/urn-3:HUL.InstRepos:dash.current.terms-of-use\#LAA

\section{Share Your Story}

The Harvard community has made this article openly available.

Please share how this access benefits you. Submit a story.

Accessibility 


\title{
RESONANT POST-NEWTONIAN ECCENTRICITY EXCITATION IN HIERARCHICAL THREE-BODY SYSTEMS
}

\author{
Smadar NaOz ${ }^{1,2,4}$, Bence Kocsis $^{1}$, Abraham Loeb $^{1}$, And Nicolás Yunes ${ }^{3}$ \\ ${ }^{1}$ Institute for Theory and Computation, Harvard-Smithsonian Center for Astrophysics, 60 Garden Street, Cambridge, MA 02138, USA; snaoz@cfa.harvard.edu \\ ${ }^{2}$ Center for Interdisciplinary Exploration and Research in Astrophysics (CIERA), Northwestern University, Evanston, IL 60208, USA \\ ${ }^{3}$ Department of Physics, Montana State University, Bozeman, MT 59718, USA \\ Received 2012 June 18; accepted 2013 July 9; published 2013 August 6
}

\begin{abstract}
We study the secular, hierarchical three-body problem to first-order in a post-Newtonian expansion of general relativity (GR). We expand the first-order post-Newtonian Hamiltonian to leading-order in the ratio of the semimajor axis of the two orbits. In addition to the well-known terms that correspond to the GR precession of the inner and outer orbits, we find a new secular post-Newtonian interaction term that can affect the long-term evolution of the triple. We explore the parameter space for highly inclined and eccentric systems, where the Kozai-Lidov mechanism can produce large-amplitude oscillations in the eccentricities. The standard lore, i.e., that GR effects suppress eccentricity, is only consistent with the parts of phase space where the GR timescales are several orders of magnitude shorter than the secular Newtonian one. In other parts of phase space, however, post-Newtonian corrections combined with the three-body ones can excite eccentricities. In particular, for systems where the GR timescale is comparable to the secular Newtonian timescales, the three-body interactions give rise to a resonant-like eccentricity excitation. Furthermore, for triples with a comparable-mass inner binary, where the eccentric Kozai-Lidov mechanism is suppressed, post-Newtonian corrections can further increase the eccentricity and lead to orbital flips even when the timescale of the former is much longer than the timescale of the secular Kozai-Lidov quadrupole perturbations.
\end{abstract}

Key words: pulsars: general - stars: evolution - stars: kinematics and dynamics

Online-only material: color figures

\section{INTRODUCTION}

Triple stellar systems are believed to be very common in nature (e.g., Tokovinin 1997; Eggleton et al. 2007). From dynamical stability arguments, these systems must be hierarchical triples, in which the (inner) binary is orbited by a third body on a much wider orbit. Probably, more than 50\% of the bright stars we see are at least (double) binary systems (Tokovinin 1997; Eggleton et al. 2007). Given the selection effects against finding faint and distant companions, we can be reasonably confident that the number of triple systems is actually substantially greater than that observed. Tokovinin (1997) showed that $40 \%$ of binary stellar systems with period $<10$ days, in which the primary is a dwarf $\left(0.5-1.5 M_{\odot}\right)$, have at least one additional companion. $\mathrm{He}$ also found that the fraction of triples and higher multiples among binaries with period (10-100 days) is $\sim 10 \%$. Moreover, Pribulla \& Rucinski (2006) have surveyed a sample of contact binaries, and noted that $42 \pm 5 \%$ of 151 of them brighter than 10 mag are at least triples. We can then conclude that many close stellar binaries with two compact objects are likely produced through triple evolution.

Long-term stability of triple system requires hierarchical configurations: an "inner" binary (with masses $m_{1}$ and $m_{2}$ ) in a nearly Keplerian orbit with semi-major axis (SMA) $a_{1}$, and an "outer" binary in which $m_{3}$ orbits the center of mass of the inner binary, with SMA $a_{2} \gg a_{1}$. Another stability condition is that the perturber does not make close approaches to the inner binary orbit. In this stability regime, a highly inclined perturber can produce large-amplitude oscillations in the eccentricity and

\footnotetext{
4 Einstein Fellow
}

inclination of the system, the so-called Kozai-Lidov mechanism (Kozai 1962; Lidov 1962).

The Kozai-Lidov mechanism is an important example of a secular effect (i.e., coherent interaction on timescales long compared to the orbital period) that is common in hierarchical triple systems but absent from two-body dynamics. This process has been proposed as an important element in the evolution of triple stars (e.g., Harrington 1969; Mazeh \& Shaham 1979; Kiseleva et al. 1998; Fabrycky \& Tremaine 2007; Perets \& Fabrycky 2009; Thompson 2011; Naoz et al. 2013; Prodan \& Murray 2012; Sharpee \& Thompson 2013) and extrasolar planetary systems with an additional distant stellar companion (e.g., Holman et al. 1997; Fabrycky \& Tremaine 2007; Wu et al. 2007; Takeda et al. 2008; Naoz et al. 2012). In addition, the Kozai-Lidov mechanism has been suggested to play an important role in both the growth of black holes (BHs) at the centers of dense stellar clusters and the formation of short-period binary BHs (Wen 2003; Miller \& Hamilton 2002). Furthermore, Ivanova et al. (2010) showed that the most important formation mechanism for $\mathrm{BH} \mathrm{X}$-ray binaries in globular clusters may be triple-induced mass transfer in a BH-white-dwarf binary.

Given the hierarchical galaxy formation paradigm, and the strong evidence that a high abundance of the local galaxies host supermassive BHs (SMBHs), one expects that major galaxy mergers should inevitably result in the formation of SMBH binaries or multiples (Valtonen 1996; Hoffman \& Loeb 2007; Kulkarni \& Loeb 2012; Dotti et al. 2012). Blaes et al. (2002) showed that the Kozai-Lidov mechanism plays an important role in the evolution of SMBH triples, where high eccentricity induced by the outer perturber can lead to a more efficient merger rate, due to gravitational wave (GW) emission (see also Seto 
2012). Also, recently Antonini \& Perets (2012) showed that secular three-body effects play an important role in the evolution of binary compact objects near SMBHs.

GWs emitted during Kozai-Lidov-induced, highly eccentric orbits of compact binaries might be detectable using $\mathrm{LIGO}^{5}$ and VIRGO $^{6}$ (e.g., Wen 2003 but see Mandel et al. 2008 and O'Leary et al. 2006), pulsar timing arrays (e.g., Finn \& Lommen 2010; Amaro-Seoane et al. 2010; Kocsis et al. 2012), and future space-based GW observatories, such as eLISA/NGO (Amaro-Seoane et al. 2012, 2012b). ${ }^{7}$ In fact, GWs associated with eccentric orbits are stronger and have a very different spectrum relative to their circular counterparts for sources at the same distance and with the same mass and spin. This may allow for the GW detection of eccentric inspirals with higher masses, larger SMAs or farther away from Earth relative to their quasi-circular counterparts (Arun et al. 2007a, 2007b; Yunes et al. 2009; O'Leary et al. 2009; Kocsis \& Levin 2012). Using GW information emitted by the close binary, it might be possible to constrain the parameters of the third body, such as its mass or distance, provided that the GW signal-to-noise ratio is sufficiently high (Yunes et al. 2011; Galaviz \& Brügmann 2011).

The Kozai-Lidov mechanism is therefore tremendously important and there is still much to be understood. Recently, Naoz et al. $(2011,2013)$ showed that an eccentric outer orbit (and even a circular one with comparable mass inner binary) can behave significantly differently than previously assumed, the so-called “eccentric Kozai-Lidov mechanism." Specifically, they showed that the inner orbits can flip from prograde to retrograde and back, and can also reach extremely high eccentricities close to unity, and the system behaves chaotically (Lithwick \& Naoz 2011). Most previous secular three-body dynamics studies that incorporated general relativity (GR) effects did so through a pseudo-potential, constructed mainly to model accretion disks and 1st post-Newtonian (1PN) shifts in the innermost stable circular orbit (Nowak \& Wagoner 1991; Artemova et al. 1996; Miller \& Hamilton 2002). It has been shown that the 1PN precession of the inner body may play an important role in secular evolution (e.g., Ford et al. 2000b; Miller \& Hamilton 2002; Blaes et al. 2002; Mardling 2007; Fabrycky \& Tremaine 2007; Zhang et al. 2013). Here we expand our investigation to include both the eccentric Kozai-Lidov mechanism and the three-body $1 P N$ effects. We show here (Section 3 and Appendix A) that although this pseudo-potential does capture some 1PN effects, such as the precession rate, the full 1PN three-body Hamiltonian introduces other corrections that cannot be modeled with this potential.

In this paper, we study the consistent inclusion of 1PN terms in the secular dynamical evolution of hierarchical triple systems. We restrict attention to the 1PN approximation of the three-body Hamiltonian. While it is well established that the eccentricity and inclination are constant in the 1PN two-body problem (Damour \& Deruelle 1985), it is not true for hierarchical triples. In addition to the standard GR precession of the inner and outer orbits, the 1PN corrections lead to a new secular interaction between the inner and outer binaries that affects their long-term evolution. We find that the standard lore, i.e., that GR effects suppress eccentricity, is only true when the GR timescales are several orders of magnitude shorter than the secular Newtonian ones. When the GR timescales are comparable to the secular

\footnotetext{
5 http://www.ligo.caltech.edu/

6 http://www.ego-gw.it/

7 http://elisa-ngo.org/
}

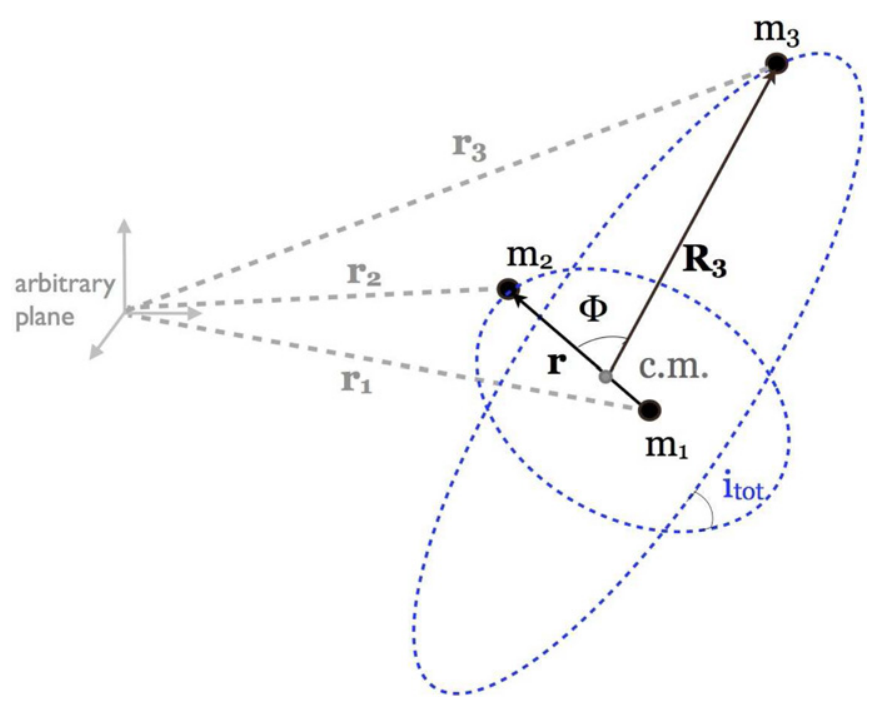

Figure 1. Coordinate system used to describe the hierarchical triple system (not to scale). Here "c.m." denotes the center of mass of the inner binary, containing objects of masses $m_{1}$ and $m_{2}$. The separation vector $\mathbf{r}$ points from $m_{1}$ to $m_{2}$; $\mathbf{R}_{3}$ points from "c.m." to $m_{3}$. The angle between the vectors $\mathbf{r}$ and $\mathbf{R}_{3}$ is $\Phi$. The distances from the bodies to a field point are labeled by $\mathbf{r}_{\mathbf{1}}, \mathbf{r}_{\mathbf{2}}$, and $\mathbf{r}_{\mathbf{3}}$.

(A color version of this figure is available in the online journal.)

Newtonian ones, we show that three-body interactions generally give rise to a resonant-like eccentricity excitation (see also Ford et al. 2000b). We will be using the term "resonance" here to describe the rapid excitations of the inner orbit's eccentricity, which occurs when the 1PN timescales are comparable to the secular Newtonian timescales. We demonstrate that even for systems with comparable inner binary masses, where the Kozai-Lidov mechanism is suppressed, and even when the GR timescales are much longer than the secular Newtonian ones, 1PN corrections continue to excite the eccentricity.

This paper is organized as follows. We begin with a definition of the parameters used to describe a hierarchical triple system based on Newtonian and 1PN three-body Hamiltonians (Section 2). We then show that three-body evolution is modified by $1 \mathrm{PN}$ effects (Section 3 ). We discuss the different timescales corresponding to the 1PN effects, and identify the region in phase space where important deviations might arise due to these terms (Section 4). We then show that 1PN terms can, in many cases, excite the eccentricity of the inner orbit instead of suppressing it (Section 5). We conclude with a discussion in Section 6.

\section{HAMILTONIAN PERTURBATION THEORY FOR HIERARCHICAL TRIPLE SYSTEMS}

A triple system consists of a binary (with masses $m_{1}$ and $m_{2}$ ) and a third body (with mass $m_{3}$ ) in orbit about the center of mass of the former. It is convenient to describe the orbits using Jacobi coordinates (Murray \& Dermott 2000). Let $\mathbf{r}$ be the relative position vector from $m_{1}$ to $m_{2}$ and $\mathbf{R}_{3}$ be the position vector of $m_{3}$ relative to the center of mass of the inner binary (see for more details Naoz et al. 2013), as shown in Figure 1.

In the PN approximation, corrections to Newtonian mechanics arise in powers of $(v / c)^{n}$, where $v$ is the orbital velocity and $c$ is the speed of light, with $n \geqslant 2$ an integer. Here we concentrate on the 1PN order corrections to Newtonian motion, which are $\mathcal{O}\left(v^{2} / c^{2}\right)$ relatively smaller than the Newtonian terms. The Hamiltonian can then be divided into a Newtonian part $\left(\mathcal{H}_{\mathrm{N}}\right)$ 
and a $1 \mathrm{PN} \operatorname{part}\left(\mathcal{H}_{1 \mathrm{PN}}\right)$ :

$$
\mathcal{H}_{\text {tot }, 1 \mathrm{PN}}=\mathcal{H}_{\mathrm{N}}+\mathcal{H}_{1 \mathrm{PN}},
$$

where the Newtonian part is simply

$$
\mathcal{H}_{\mathrm{N}}=\frac{1}{2} \sum_{i=1}^{3} \frac{p_{i}^{2}}{m_{i}}-\frac{1}{2} \sum_{i, j \neq i}^{3} \frac{k^{2} m_{i} m_{j}}{r_{i j}}
$$

and the 1PN part is (e.g., Schäfer 1987; Moore 1993; Lousto \& Nakano 2008):

$$
\begin{aligned}
\mathcal{H}_{1 \mathrm{PN}}= & -\frac{1}{8 c^{2}} \sum_{i=1}^{3} m_{i}\left(\frac{p_{i}^{2}}{m_{i}^{2}}\right)^{2}-\frac{k^{2}}{4 c^{2}} \sum_{i, i \neq j} \frac{m_{i} m_{j}}{r_{i j}} \\
& \times\left\{6 \frac{p_{i}^{2}}{m_{i}^{2}}-7 \frac{\left(\mathbf{p}_{i} \cdot \mathbf{p}_{j}\right)}{m_{i} m_{j}}-\frac{\left(\mathbf{n}_{i j} \cdot \mathbf{p}_{i}\right)\left(\mathbf{n}_{i j} \cdot \mathbf{p}_{j}\right)}{m_{i} m_{j}}\right\} \\
& +\frac{k^{4}}{2 c^{2}} \sum_{i, j \neq i, k^{\prime} \neq i} \frac{m_{i} m_{j} m_{k^{\prime}}}{r_{i j} r_{i k^{\prime}}} .
\end{aligned}
$$

In these equations, $k^{2}$ is the gravitational constant, $\mathbf{r}_{\mathbf{i j}}\left(r_{i j}\right)$ is the relative position vector (magnitude) from mass $m_{i}$ to $m_{j}$, $\mathbf{p}_{i}\left(p_{i}\right)$ is the momentum vector (magnitude) of mass $m_{i}$ in an arbitrary plane (we shall later transform to center-of-mass coordinates). In the 1PN Hamiltonian, $i, j$ and $k^{\prime}$ run from 1 to 3 (the three masses), where $k^{\prime}$ is an index while $k^{2}$ is the gravitational constant, and $\mathbf{n}_{i j}=\mathbf{r}_{i j} / r_{i j}$.

Many gravitational triple systems are in a hierarchical configuration: two objects orbit each other in a relatively tight inner binary while the third object is on a much wider orbit. If the third object is sufficiently distant, an analytic, perturbative approach can be used to calculate the evolution of the system over long timescales (relative to the orbital period). In the usual secular approximation (e.g., Marchal 1990), the three orbiting objects torque each other and exchange angular momentum, but not energy. Therefore, on timescales much longer than their orbital periods, the eccentricity and orientation can change, but not the SMA.

Given this, the orbital motion of a triple system can be divided into two separate Keplerian orbits: the relative orbit of bodies 1 and 2, and the orbit of body 3 around the center of mass of the system. The Hamiltonian for the system can then be decomposed accordingly into two Keplerian Hamiltonians plus a coupling term that describes the (weak) interaction between the two orbits. Let the SMAs of the inner and outer orbits be $a_{1}$ and $a_{2}$, respectively. Then, the coupling term in the Hamiltonian can be written as a power series in the ratio of the SMAs $\alpha=a_{1} / a_{2}$ (e.g., Harrington 1968). In a hierarchical system, by definition, this parameter $\alpha$ is small.

The Newtonian part of the Hamiltonian, expanded in powers of $\alpha$, is (e.g., Harrington 1968)

$$
\begin{aligned}
\mathcal{H}_{\mathrm{N}}= & -\frac{k^{2} m_{1} m_{2}}{2 a_{1}}-\frac{k^{2} m_{3}\left(m_{1}+m_{2}\right)}{2 a_{2}} \\
& -\frac{k^{2}}{a_{2}} \sum_{j=2}^{\infty} \alpha^{j} M_{j}\left(\frac{r}{a_{1}}\right)^{j}\left(\frac{a_{2}}{R_{3}}\right)^{j+1} P_{j}(\cos \Phi),
\end{aligned}
$$

where $P_{j}$ are Legendre polynomials, $\Phi$ is the angle between $\mathbf{r}_{1}$ and $\mathbf{r}_{2}$ (see Figure 1), and

$$
M_{j}=m_{1} m_{2} m_{3} \frac{m_{1}^{j-1}-\left(-m_{2}\right)^{j-1}}{\left(m_{1}+m_{2}\right)^{j}} .
$$

Note that most secular studies follow the convention of Harrington (1969) and choose the Hamiltonian to be the negative of the total energy, so that $\mathcal{H}>0$ for bound systems. Here we did not follow this convention. The equations of motion in Naoz et al. (2013) did use this convention, and thus, a reader that wishes to combine the two sets of equations need to introduce a minus sign to one of the sets.

We adopt canonical variables, known as Delaunay's elements, which provide a particularly convenient dynamical description of hierarchical three-body systems (e.g., Valtonen \& Karttunen 2006). The coordinates are chosen to be the mean anomalies, $l_{1}$ and $l_{2}$, the arguments of periastron, $g_{1}$ and $g_{2}$, and the longitudes of ascending nodes, $h_{1}$ and $h_{2}$, where subscripts 1,2 denote the inner and outer orbits, respectively. Their Newtonian conjugate momenta are

$$
\begin{aligned}
& L_{1}=\frac{m_{1} m_{2}}{m_{1}+m_{2}} \sqrt{k^{2}\left(m_{1}+m_{2}\right) a_{1}}, \\
& L_{2}=\frac{m_{3}\left(m_{1}+m_{2}\right)}{m_{1}+m_{2}+m_{3}} \sqrt{k^{2}\left(m_{1}+m_{2}+m_{3}\right) a_{2}}, \\
& G_{1}=L_{1} \sqrt{1-e_{1}^{2}}, \quad G_{2}=L_{2} \sqrt{1-e_{2}^{2}},
\end{aligned}
$$

and

$$
H_{1}=G_{1} \cos i_{1}, \quad H_{2}=G_{2} \cos i_{2},
$$

respectively, where $e_{1}\left(e_{2}\right)$ is the inner (outer) orbital eccentricity. Note that $G_{1}$ and $G_{2}$ are also the magnitudes of the angular momentum vectors $\left(\mathbf{G}_{1}\right.$ and $\left.\mathbf{G}_{2}\right)$, and $H_{1}$ and $H_{2}$ are the $z$-components of these vectors. The following geometric relations between the momenta follow from the law of cosines:

$$
\begin{aligned}
\cos i_{\mathrm{tot}} & =\frac{G_{\mathrm{tot}}^{2}-G_{1}^{2}-G_{2}^{2}}{2 G_{1} G_{2}}, \\
H_{1} & =\frac{G_{\mathrm{tot}}^{2}+G_{1}^{2}-G_{2}^{2}}{2 G_{\mathrm{tot}}}, \\
H_{2} & =\frac{G_{\mathrm{tot}}^{2}+G_{2}^{2}-G_{1}^{2}}{2 G_{\mathrm{tot}}},
\end{aligned}
$$

where $\mathbf{G}_{\mathrm{tot}}=\mathbf{G}_{1}+\mathbf{G}_{2}$ is the (conserved) total angular momentum vector, and the angle between $\mathbf{G}_{\mathbf{1}}$ and $\mathbf{G}_{\mathbf{2}}$ defines the mutual inclination $i_{\text {tot }}=i_{1}+i_{2}$. From Equations (10) and (11) we find that the inclinations $i_{1}$ and $i_{2}$ are determined by the orbital angular momenta via

$$
\begin{aligned}
& \cos i_{1}=\frac{G_{\mathrm{tot}}^{2}+G_{1}^{2}-G_{2}^{2}}{2 G_{\mathrm{tot}} G_{1}}, \\
& \cos i_{2}=\frac{G_{\mathrm{tot}}^{2}+G_{2}^{2}-G_{1}^{2}}{2 G_{\mathrm{tot}} G_{2}} .
\end{aligned}
$$

In addition to these geometrical relations we also have that

$$
H_{1}+H_{2}=G_{\text {tot }}=\text { constant }
$$

since we are here neglecting dissipative effects such as GW radiation-reaction, and thus, the Hamiltonian is conserved. Given this parameterization, the Hamiltonian or canonical 
equations describe the orbital motion via

$$
\begin{aligned}
\frac{d L_{j}}{d t}=-\frac{\partial \mathcal{H}}{\partial l_{j}}, & \frac{d l_{j}}{d t}=\frac{\partial \mathcal{H}}{\partial L_{j}}, \\
\frac{d G_{j}}{d t}=-\frac{\partial \mathcal{H}}{\partial g_{j}}, & \frac{d g_{j}}{d t}=\frac{\partial \mathcal{H}}{\partial G_{j}}, \\
\frac{d H_{j}}{d t}=-\frac{\partial \mathcal{H}}{\partial h_{j}}, & \frac{d h_{j}}{d t}=\frac{\partial \mathcal{H}}{\partial H_{j}},
\end{aligned}
$$

where $j=1,2$.

The secular Hamiltonian (both the Newtonian and the 1PN parts) is given by taking Equation (1) expanded in powers of $\alpha$ and averaging over the rapidly varying $l_{1}$ and $l_{2}$. The averaging technique we use is known as the Von Zeipel transformation (for more details, see Brouwer 1959; Naoz et al. 2013; also see Appendix B) a canonical transformation that eliminates the rapidly-oscillating parts of $\mathcal{H}$. We apply this transformation twice, leading to a Hamiltonian that is the double average of the original Hamiltonian over both orbital periods. We thus refer to the resulting quantity as the "double-averaged Hamiltonian."

The double-averaged Newtonian Hamiltonian (Equation (4)), up to octupole order (i.e., up to $\mathcal{O}\left(\alpha^{3}\right)$ beyond the leading order term proportional to $a_{1}^{-1}$, see Naoz et al. 2013) ${ }^{8}$ can be written as

$$
\overline{\mathcal{H}}_{\mathrm{N}}=\overline{\mathcal{H}}_{\text {quad }}^{\mathrm{N}}+\overline{\mathcal{H}}_{\mathrm{oct}}^{\mathrm{N}}
$$

where

$$
\begin{aligned}
\overline{\mathcal{H}}_{\text {quad }}^{\mathrm{N}}= & -C_{2}\left\{\left(2+3 e_{1}^{2}\right)\left(3 \cos ^{2} i_{\text {tot }}-1\right)\right. \\
& \left.+15 e_{1}^{2} \sin ^{2} i_{\text {tot }} \cos \left(2 g_{1}\right)\right\}, \\
\overline{\mathcal{H}}_{\mathrm{oct}}^{\mathrm{N}}= & \frac{15}{4} \epsilon_{M} e_{1} C_{2}\left\{A \cos \phi+10 \cos i_{\text {tot }} \sin ^{2} i_{\text {tot }}\right. \\
& \left.\times\left(1-e_{1}^{2}\right) \sin g_{1} \sin g_{2}\right\} .
\end{aligned}
$$

Note that Equation (19) has a minus sign compared to Naoz et al. (2013), which used the sign convention for which the Hamiltonian is positive. Here, we did not include the terms which correspond to the Keplerian orbital energy of the three objects which depend on only the SMAs, and are constant in the secular approximation without dissipative effects. Furthermore, we have defined

$$
\begin{aligned}
\epsilon_{M} & =\left(\frac{m_{1}-m_{2}}{m_{1}+m_{2}}\right)\left(\frac{a_{1}}{a_{2}}\right) \frac{e_{2}}{1-e_{2}^{2}}, \\
C_{2} & =\frac{k^{4}}{16} \frac{\left(m_{1}+m_{2}\right)^{7}}{\left(m_{1}+m_{2}+m_{3}\right)^{3}} \frac{m_{3}^{7}}{\left(m_{1} m_{2}\right)^{3}} \frac{L_{1}^{4}}{L_{2}^{3} G_{2}^{3}}, \\
A & =4+3 e_{1}^{2}-\frac{5}{2} B \sin i_{\text {tot }}^{2}, \\
B & =2+5 e_{1}^{2}-7 e_{1}^{2} \cos \left(2 g_{1}\right),
\end{aligned}
$$

\footnotetext{
8 Note that Krymolowski \& Mazeh (1999) showed that the von Zeipel transformation results in higher orders terms proportional to $\alpha^{7 / 2}$; however, here we consider only $\mathcal{O}\left(\alpha^{3}\right)$ level of perturbations.
}

and

$$
\cos \phi=-\cos g_{1} \cos g_{2}-\cos i_{\text {tot }} \sin g_{1} \sin g_{2} .
$$

Note that the octupole coefficient in Ford et al. (2000b), is simply $C_{3}=C_{2}\left(\epsilon_{M} / e_{2}\right) 15 / 4$. Also, following our definitions (see Figure 1) $m_{1}$ and $m_{2}$ refer to the component masses of the inner orbit, while $e_{2}$ refers to the eccentricity of the outer orbit. In the test-particle limit (i.e., $\left.m_{1} \gg m_{2}\right) \epsilon_{M}$ (Equation (21)) reduces to the octupole coefficient introduced in Lithwick \& Naoz (2011) and Katz et al. (2011),

$$
\epsilon=\left(\frac{a_{1}}{a_{2}}\right) \frac{e_{2}}{1-e_{2}^{2}} .
$$

In these Hamiltonians (and in the following 1PN parts), we have eliminated the nodes (i.e., $h_{1}$ and $h_{2}$ ) by using the conservation of total angular momentum, which leads to $h_{1}-h_{2}=\pi$. As shown in Naoz et al. (2013) this can be done only as long as one does not conclude that the conjugate momenta are constant (e.g., Dirac 1950). The full equations of motion up to the Newtonian octupole order are presented in Naoz et al. (2013).

The averaged 1PN Hamiltonian can be separated into different terms. First, let us use the fact that for Keplerian orbits the momentum can be related to the radius and SMA; for the inner orbit, we can write $p_{\text {in }}=\mu_{\text {in }} \sqrt{k^{2}\left(m_{1}+m_{2}\right)\left(2 / r-1 / a_{1}\right)}$, where $\mu_{\text {in }}$ is the reduced mass of the inner orbit and a similar relation can be written to the outer orbit. Second, we substitute this relation into the three-body 1PN Hamiltonian, i.e., Equation (3). After transforming to the center of mass frame, the 1PN corrections is expanded in powers of $\alpha$ up to relative $\mathcal{O}\left(\alpha^{3}\right)$. This produces a similar expansion to Equation (4) for the 1PN Hamiltonian, but due to its length we have chosen not to present it here. To investigate the long-term dynamics of the three-body system, we eliminate all terms with short periods in the Hamiltonian, which depend on the rapidly changing $l_{1}$ and $l_{2}$, using a double Von Zeipel transformation (Brouwer 1959), see for more details Appendix B. In doing so, we must first calculate the angle between the vectors $\mathbf{p}_{\text {in }} \cdot \mathbf{p}_{\text {out }}$ and $\mathbf{p}_{\text {out }} \cdot \mathbf{r}$ and $\mathbf{p}_{\text {in }} \cdot \mathbf{R}_{3}$, where $\mathbf{p}_{\text {in }}$ ( $\mathbf{p}_{\text {out }}$ ) is the momentum of the inner (outer) orbit, as defined in the invariable plane.

The leading-order term in an $\alpha \ll 1$ expansion is proportional to $a_{1}^{-2}$ in the double-averaged 1PN Hamiltonian. Keeping all terms up to $\mathcal{O}\left(\alpha^{3}\right)$ beyond leading gives

$$
\overline{\mathcal{H}}^{1 \mathrm{PN}}=\overline{\mathcal{H}}_{a_{1}^{-2}}^{1 \mathrm{PN}}+\overline{\mathcal{H}}_{a_{1} a_{2}}^{1 \mathrm{PN}}+\overline{\mathcal{H}}_{a_{2}^{-2}}^{1 \mathrm{PN}} \overline{+} \mathcal{H}_{\mathrm{int}}^{1 \mathrm{PN}}
$$

where

$$
\begin{aligned}
\overline{\mathcal{H}}_{a_{1}^{-2}}^{1 \mathrm{PN}}= & \frac{k^{4} \mu_{\text {in }}\left(15 m_{1}^{2}+29 m_{1} m_{2}+15 m_{2}^{2}\right)}{8 a_{1}^{2} c^{2}} \\
& -\frac{3 k^{4} m_{1} m_{2}\left(m_{1}+m_{2}\right)}{a_{1}^{2} c^{2} \sqrt{1-e_{1}^{2}}} \\
\overline{\mathcal{H}}_{a_{1} a_{2}}^{1 \mathrm{PN}}= & \frac{k^{4} m_{1} m_{2} m_{3}\left(2\left(m_{1}+m_{2}\right)+3 m_{3}\right)}{4 a_{1} a_{2} c^{2}\left(m_{1}+m_{2}+m_{3}\right)}, \\
\overline{\mathcal{H}}_{a_{2}^{-2}}^{1 \mathrm{PN}}= & \frac{k^{4} \mu_{\mathrm{out}}\left(15\left(m_{1}+m_{2}\right)^{2}+29\left(m_{1}+m_{2}\right) m_{3}+15 m_{3}^{2}\right)}{8 a_{2}^{2} c^{2}\left(m_{1}+m_{2}+m_{3}\right)} \\
& -\frac{3 k^{4}\left(m_{1}+m_{2}\right) m_{3}\left(m_{1}+m_{2}+m_{3}\right)}{a_{2}{ }^{2} c^{2} \sqrt{1-e_{2}^{2}}}
\end{aligned}
$$




$$
\begin{aligned}
\overline{\mathcal{H}}_{\text {int }}^{1 \mathrm{PN}}= & \frac{k^{2}}{4 a_{2}^{3} c^{2}\left(1-e_{2}^{2}\right)^{3 / 2}\left(m_{1}+m_{2}\right)}\left\{G _ { 1 } G _ { 2 } \left[8\left(m_{1}+m_{2}\right)\right.\right. \\
& \left.+6 m_{3}\right] \cos i_{\text {tot }}+\frac{a_{1} k^{2} m_{1} m_{2} m_{3}}{8\left(m_{1}+m_{2}\right)}\left(f_{m e_{1}}-3 f_{m e_{1}} \cos ^{2} i_{\text {tot }}\right. \\
& \left.\left.+9 e_{1}^{2}\left(m_{1}^{2}+m_{1} m_{2}+m_{2}^{2}\right) \cos \left(2 g_{1}\right) \sin ^{2} i_{\text {tot }}\right)\right\}
\end{aligned}
$$

and where

$$
\begin{aligned}
\mu_{\text {in }} & =\frac{m_{1} m_{2}}{m_{1}+m_{2}}, \\
\mu_{\text {out }} & =\frac{m_{3}\left(m_{1}+m_{2}\right)}{m_{1}+m_{2}+m_{3}}, \\
f_{m e_{1}} & =\left(2-5 e_{1}^{2}\right)\left(m_{1}^{2}+m_{2}^{2}\right)-3\left(2-e_{1}^{2}\right) m_{1} m_{2} .
\end{aligned}
$$

Here $\overline{\mathcal{H}}_{\text {int }}^{1 \mathrm{PN}}$ includes all terms of $\mathcal{O}\left(\alpha^{5 / 2}\right)$ and $\mathcal{O}\left(\alpha^{3}\right)$ beyond $\overline{\mathcal{H}}_{a_{1}^{-2}}^{1 \mathrm{PN}}$, since $G_{1} \propto a_{1}^{1 / 2}$ and $G_{2} \propto a_{2}^{1 / 2}$ according to Equations (6) and (7).

Not all of the different 1PN Hamiltonian terms affect the dynamical evolution of the triple. The $\overline{\mathcal{H}}_{a_{1} a_{2}}^{1 \mathrm{PN}}$ term only depends on the masses and the SMAs, i.e., it does not depend on the canonical coordinates, and thus, it does not affect the canonical equations, although it does change the total energy of the system. On the other hand, $\overline{\mathcal{H}}_{a_{1}^{-2}}^{1 \mathrm{PN}}$ and $\overline{\mathcal{H}}_{a_{2}^{-2}}^{1 \mathrm{PN}}$ do contribute to the dynamical evolution, as they clearly depend on $e_{1}$ and $e_{2}$. A possible, (intuitive) physical explanation for this is the following. In the $a_{2} \rightarrow \infty$ limit, one would expect only two physical effects: precession of the inner orbit and precession of the outer orbit about the inner binary. These two physical effects arise because of $\overline{\mathcal{H}}_{a_{1}^{-2}}^{1 \mathrm{PN}}$ and $\overline{\mathcal{H}}_{a_{2}^{-2}}^{1 \mathrm{PN}}$, and thus, $\overline{\mathcal{H}}_{a_{1} a_{2}}^{1 \mathrm{PN}}$, (which satisfies $\overline{\mathcal{H}}_{a_{1} a_{2}}^{1 \mathrm{PN}} \gg \overline{\mathcal{H}}_{a_{2}^{-2}}^{1 \mathrm{PN}}$ for large $\left.a_{2} / a_{1}\right)$ cannot contribute to the motion.

The quantity $\overline{\mathcal{H}}_{\text {int }}^{1 \mathrm{PN}}$ is an "interaction term," in that it represents the coupling between the outer and the inner orbits. Notice that this term would not be present if we had truncated the $\alpha \ll 1$ expansion at $\mathcal{O}\left(\alpha^{2}\right)$. Notice also that the interaction term does not depend on the argument of periapsis of the outer orbit, $g_{2}$, just like the quadrupole Newtonian Hamiltonian. Therefore, at quadrupole order, e.g., for a circular outer perturber, the absence of $g_{2}$ in the Hamiltonian implies that the outer orbital angular momentum, $G_{2}$, is conserved (the so-called "happy coincidence" of Lidov \& Ziglin 1976).

\section{TRIPLE BODY EVOLUTION IN POST-NEWTONIAN THEORY}

The secular evolution of a three-body hierarchical system to Newtonian, octupole order was studied in Naoz et al. (2013). As mentioned in Section 1, they showed that the commonly assumed conservation of the $z$-component of the angular momenta of the inner and outer orbits $\left(H_{1}\right.$ and $\left.H_{2}\right)$ is only correct in the test-particle approximation to quadrupole order. Newtonian octupole terms further modulate the eccentricity and inclination oscillations. Specifically, for an eccentric and inclined outer perturber, these terms can lead to extremely high eccentricities and flip the inner orbit from prograde to retrograde. This type of behavior also appears in the test-particle limit for an eccentric orbit (e.g., Lithwick \& Naoz 2011; Katz et al. 2011; Naoz et al. 2013).

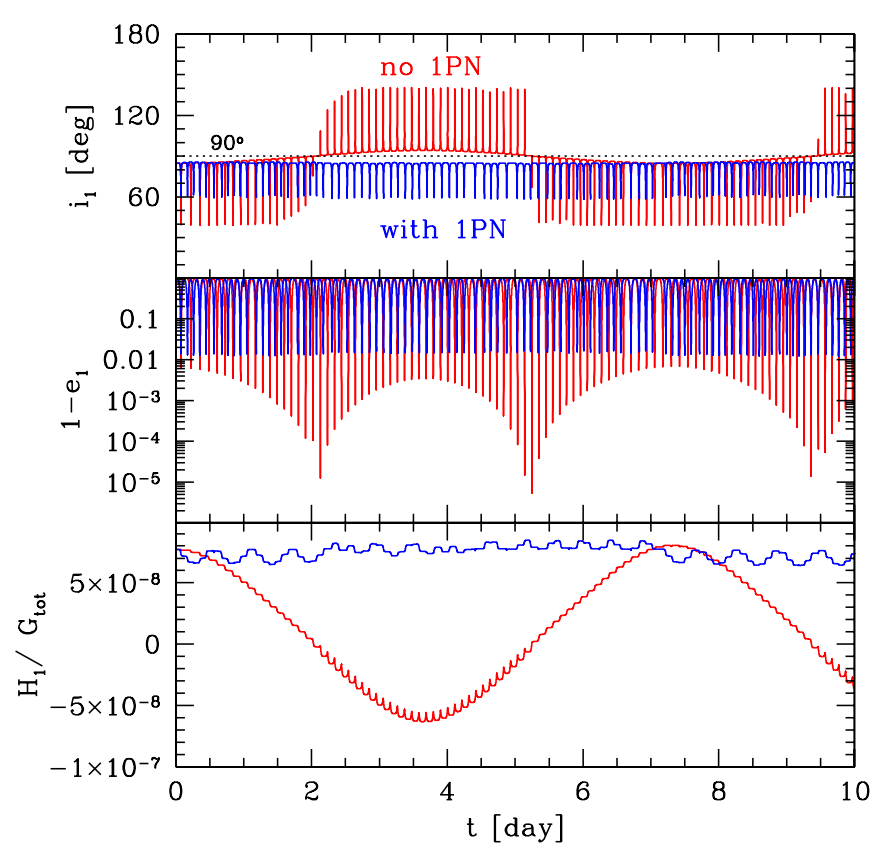

Figure 2. An example of the evolution of a triple to Newtonian octupole order neglecting PN contributions (red lines) and including them up to the $1 \mathrm{PN}$ interactions terms in the double-averaged Hamiltonian (blue lines). The system has an inner binary of $m_{1}=1 M_{\odot}, m_{2}=0.001 M_{\odot}$, and the third object mass is $m_{3}=10^{4} M_{\odot}$. We set $a_{1} / R_{1}^{g}=10^{4}$ and $a_{2} / R_{3}^{g}=202$. We set initially $e_{2}=0.6, e_{1}=0.01 g_{1}=g_{2}=0^{\circ}$ and $i_{\text {tot }}=85^{\circ}$. We consider, from top to bottom, the inclination of the inner orbit, $i_{1}$, the eccentricity of the inner orbit in terms of $1-e_{1}$, and the z-component of the angular momentum normalized to the total angular momentum. Note that we do not plot $G_{2}$ since in configuration where $m_{2} \rightarrow 0, G_{2} \rightarrow G_{\text {tot }}$, (Lithwick \& Naoz 2011). In this case, the quadrupole Newtonian terms induce eccentricity-inclination oscillations, modulated by the octupole terms, while the 1PN effect suppresses them.

(A color version of this figure is available in the online journal.)

Figure 2 presents the secular evolution of a three-body hierarchical system to Newtonian, octupole order (red lines). We chose a system with inner binary masses $m_{1}=1 M_{\odot}$ and $m_{2}=$ $0.001 M_{\odot}$, and an outer binary companion with mass $m_{3}=$ $10^{4} M_{\odot}$. For this system, we set $a_{1} / R_{1}^{g}=10^{4}$ and $a_{2} / R_{3}^{g}=202$, where, $R_{1}^{g}=k^{2}\left(m_{1}+m_{2}\right) / c^{2}$ and $R_{3}^{g}=k^{2} m_{3} / c^{2}$ are the gravitational radii of the inner and the outer orbits, respectively. We also set initially $e_{2}=0.6, e_{1}=0.01, g_{1}=g_{2}=0^{\circ}$ and $i_{\text {tot }}=85^{\circ}$. The Newtonian quadrupole terms induce the "standard" eccentricity-inclination oscillations, while octupole terms modulate it. As can be seen in the figure, the modulation does not have a precise periodicity and, in fact, the octupole terms introduce the chaotic aspects to the evolution (Lithwick \& Naoz 2011). When 1PN corrections become significant however, the evolutionary orbital tracks can be significantly different (already at quadrupole order).

Different 1PN terms have different effects on the evolutionary orbital tracks, where the perturbations to the equations of motion follow from Equations (15)-(17). $\overline{\mathcal{H}}_{a_{1}^{-2}}^{1 \mathrm{PN}}$ gives rise to the standard GR precession of the argument of periapsis of the inner orbit, while $\overline{\mathcal{H}}_{a_{2}^{-2}}^{1 \mathrm{PN}}$ is responsible for the precession of the argument of periapsis of the outer orbit,

$$
\begin{aligned}
\left.\frac{d g_{1}}{d t}\right|_{1 \mathrm{PN}\left(a_{1}^{-2}\right)} & =\frac{3 k^{3}\left(m_{1}+m_{2}\right)^{3 / 2}}{a_{1}^{5 / 2} c^{2}\left(1-e_{1}^{2}\right)}, \\
\left.\frac{d g_{2}}{d t}\right|_{1 \mathrm{PN}\left(a_{2}^{-2}\right)} & =\frac{3 k^{3}\left(m_{1}+m_{2}+m_{3}\right)^{3 / 2}}{a_{2}^{5 / 2} c^{2}\left(1-e_{2}^{2}\right)} .
\end{aligned}
$$


These contributions can be recovered independently from the individual two-body 1PN Hamiltonians of the inner and outer binary (see Appendix A Equation (A1)), or from an effective potential, or directly from the 1PN metric (e.g., Misner et al. 1973 , chap. 25 , p. $668-670)$. Other than this precession, $\overline{\mathcal{H}}_{a_{1}^{-2}}^{1 \mathrm{PN}}$ and $\overline{\mathcal{H}}_{a_{2}^{-2}}^{1 \mathrm{PN}}$, do not directly affect the other orbital elements. ${ }^{9}$ The $\overline{\mathcal{H}}_{a_{1} a_{2}}^{1 \mathrm{PN}}$ term just modifies the total energy and does not modify the long-term dynamical evolution at all, as long as dissipative effects are neglected.

In the standard lore, if the GR precession rate of the inner orbit is faster than the quadrupole secular Newtonian timescales, the GR effect is presumed to suppress the eccentricity growth (for an $m_{2}$ test particle to quadrupole Newtonian order, see Fabrycky $\&$ Tremaine 2007). In Figure 2 (blue lines) we show an example where this is indeed the case, even when including all 1PN terms (see below). In this example, eccentricity (and orbital flips) are suppressed by the 1PN corrections (the variations shown by the blue lines are shorter than red lines).

The usual precession term (Equation (35)) is not sufficient to model the system, as one must also account for the precession of the outer orbit (Equation (36)) and the other effects introduced by the $1 \mathrm{PN}$ interaction terms (Appendix C). The inclusion of these terms leads to qualitatively different behavior because they directly drive the evolution of inner and outer orbital eccentricity and inclination, while Equation (35) (Equation (36)) drives the evolution of only the argument of periapsis of the inner (outer) orbit. Figure 3 shows the evolution of the eccentricity when different terms in the Hamiltonian are included. We considered a system with parameters $m_{1}=1 M_{\odot}, m_{2}=1 M_{\mathrm{J}}$ (essentially a test particle), $m_{3}=10^{6} M_{\odot}, a_{1} / R_{1}^{g}=5.06 \times 10^{5}$ corresponding to $0.005 \mathrm{AU}$. In the left column we consider initial relative inclination $i_{\text {tot }}=95^{\circ}$ and a separation of the outer orbit $a_{2} / R_{3}^{g}=5.2 \times 10^{3}$ corresponding to $51.4 \mathrm{AU}$. In the right column, we consider initial relative inclination $i_{\text {tot }}=65^{\circ}$ and a separation of the outer orbit $a_{2} / R_{3}^{g}=4.8 \times 10^{3}$ corresponding to 47.35 AU. For the calculation in the two columns, the initial eccentricities are $e_{1}=0.001$ and $e_{2}=0.7$, and the initial argument of pericenter of the inner and outer orbits is set to $240^{\circ}$ and zero, respectively. This system configuration is such that the 1PN $\left(a_{1}^{-2}\right)$ timescales for circular orbits $(\sim 59 \mathrm{yr}$, for the left column example, see Section 4 for more details) are shorter than the Newtonian quadrupole ones $(\sim 145 \mathrm{yr}$ for the left column example). Nevertheless, the secular eccentricity oscillations are still present. The bottom panel shows that the interaction term $\overline{\mathcal{H}}_{\text {int }}^{1 \mathrm{PN}}$ introduces a significant new periodic modulation to the eccentricity evolution. We discuss in more details the conditions in parameter space that lead to this behavior in Sections 4 and 5 (note that this system represents the resonance peak of the $95^{\circ}$ and $65^{\circ}$ cases of Figure 5 below).

The usual precession term (mostly for the inner) in the presence of three-body secular evolution was compared to direct $\mathrm{N}$-body calculation in the literature before (e.g., Ford et al. 2000b; Zhang et al. 2013). To resolve the effects of the interaction term one needs to be in the regime where the GR precession timescales are much shorter than the quadrupole precession timescales (see Section 4). The examples considered in Figure 3 represent $\sim 3 \times 10^{8}$ of the inner orbital period. Numerical integrations algorithms that conserve the energy over such long timescales in the three-body post Newtonian regime

\footnotetext{
9 Note, however, that the precession indirectly affects the evolution of the
} other orbital elements through $\overline{\mathcal{H}}^{\mathrm{N}}$, as shown below.
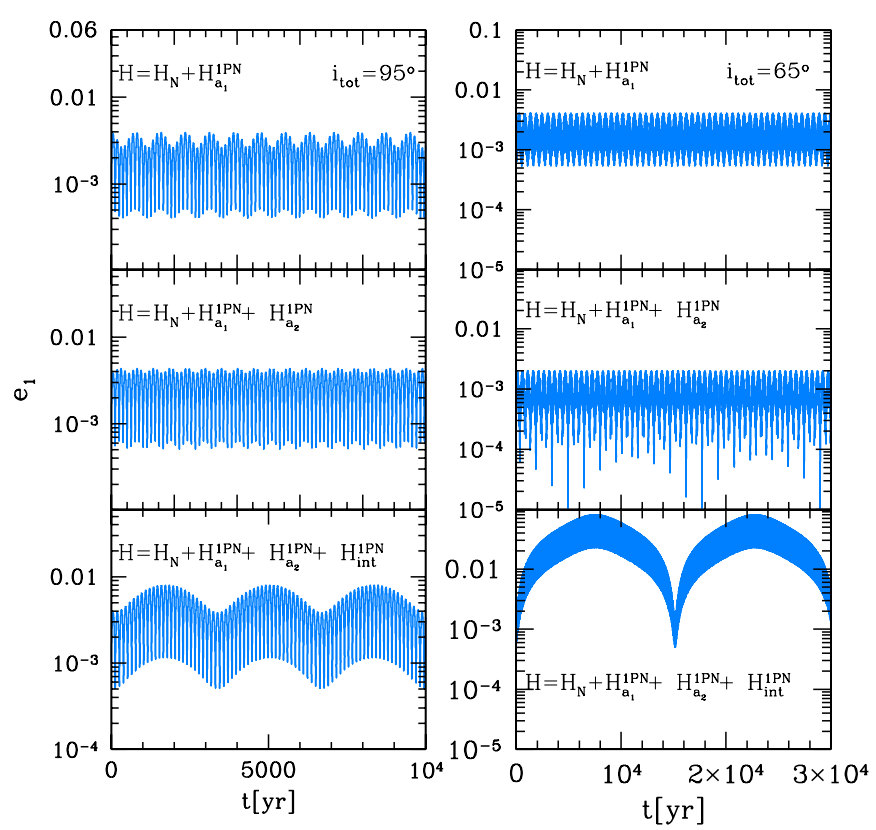

Figure 3. Two examples of the time evolution of the system dominated by 1PN effects; the right and left side panels differ only in the initial relative inclination and the outer orbital separation. In the top panels we consider the three-body orbit evolution due to Newtonian dynamics and the lowest-level 1PN correction, i.e., $\mathcal{H}_{N}+\mathcal{H}_{a_{1}^{-2}}^{1 \mathrm{PN}}$. In the middle panels we add the next level of approximation $+\mathcal{H}_{a_{2}^{-2}}^{1 \mathrm{PN}}$, and in the bottom panels we consider the approximation up to the highest level discussed here, i.e., $+\mathcal{H}_{\text {int }}^{1 \mathrm{PN}}$. The inner binary contains an object of mass $1 M_{\odot}$ and an object of mass $1 M_{\mathrm{J}}$ (can be considered as a test particle), while the outer object is a $\mathrm{BH}$ with mass of $10^{6} M_{\odot}$. The inner orbital separation is $a_{1} / R_{1}^{g}=5.06 \times 10^{5}$, corresponding to $0.005 \mathrm{AU}$. The initial eccentricities are $e_{1}=0.001$ and $e_{2}=0.7$. The arguments of pericenter of the inner orbit initially set to $240^{\circ}$ and outer orbit initially is set to zero. In the left column, we consider an initial relative inclination of $i_{\text {tot }}=95^{\circ}$, and an outer orbital separation of $a_{2} / R_{3}^{g}=5.2 \times 10^{3}$, corresponding to $51.4 \mathrm{AU}$. In the right column, we consider an initial relative inclination of $i_{\text {tot }}=65^{\circ}$, and an outer orbital separation of $a_{2} / R_{3}^{g}=4.8 \times 10^{3}$, corresponding to $47.35 \mathrm{AU}$. Observe that as one includes more $1 \mathrm{PN}$ effects, qualitatively different behavior emerges.

(A color version of this figure is available in the online journal.)

are not easy to implement or develop, and thus, they are left to future work.

One might worry that a $1 \mathrm{PN}$ treatment might not be sufficient to model certain regions of phase space, as we have neglected 2PN and higher PN order terms in the evolution. Such terms become important when the PN perturbation parameter, $v / c$ with $v$ any system velocity, i.e., the pericenter velocities $\left(v_{p 1} / c\right)^{2}=\left(R_{1}^{g}+R_{2}^{g}\right) /\left[a_{1}\left(1-e_{1}\right)\right]$ and similarly for the outer orbit, are not sufficiently small. In most of our examples we ensured that our systems are well within the PN regime, however, for very eccentric systems, $2 \mathrm{PN}$ and higher PN order terms may be important. In fact, one might naively expect the 1PN corrections accounted for here to be negligible if $v / c \ll 1$. This is not so, because although the 1PN terms are small at any point in time, their effects may accumulate and become significant over long timescales in the three-body problem.

\section{TIMESCALES}

In order to explore the regions of phase space where the 1PN effects may be expected to become significant, we compare the various timescales associated with the individual terms in the Hamiltonian.

The timescale associated with the Newtonian quadrupole term can be estimated from the canonical equations. More precisely, 
$t_{\text {quad }}^{\mathrm{N}} \sim 2 \pi G_{1} / C_{2}$, where $C_{2}$ is given in Equation (22):

$$
t_{\text {quad }}^{\mathrm{N}} \sim \frac{2 \pi a_{2}^{3}\left(1-e_{2}^{2}\right)^{3 / 2} \sqrt{m_{1}+m_{2}}}{a_{1}^{3 / 2} m_{3} k}
$$

The timescales associated with the Newtonian octupole terms are more difficult to estimate due to their chaotic effect on the orbits. For example, in Figure 2 the first modulation period is shorter than the second (see Naoz et al. 2013 for more examples). However, as demonstrated in Lithwick \& Naoz (2011), although the system is chaotic when the octupole terms are included, there are two general features in the evolution: one associated with an octupole timescale, defined below, and a shorter one (see for example their Figure 7 of surfaces of section, which shows the two different evolutionary behaviors). We define a timescale for the regular part of the Newtonian octupole evolution through the rough estimate $t_{\mathrm{oct}}^{\mathrm{N}} \sim(4 / 15) t_{\text {quad }}^{\mathrm{N}} / \epsilon_{M}$ for a given inner and outer eccentricity,

$$
t_{\mathrm{oct}}^{\mathrm{N}} \sim 2 \pi \frac{4}{15} \frac{a_{2}^{4}\left(1-e_{2}^{2}\right)^{5 / 2} \sqrt{1-e_{1}^{2}}\left(m_{1}+m_{2}\right)^{3 / 2}}{a_{1}^{5 / 2} e_{2} k\left|m_{1}-m_{2}\right| m_{3}} .
$$

Note that when the inner binary is very eccentric, these timescales can change by orders of magnitude. At octupole order, the eccentricity of the outer orbit can also oscillate, although usually these oscillations are small in magnitude. For the remainder of this section, we employ a test-particle approximation for one of the components of the inner binary, i.e., $m_{2} \rightarrow 0$, for which $e_{2}=$ const. (Lithwick \& Naoz 2011). We will see that this is sufficient to understand the regions of phase space where $1 \mathrm{PN}$ terms become important.

The 1PN timescale can be estimated as in Equation (37), which gives

$$
\begin{aligned}
& t_{a_{1}^{-2}}^{1 \mathrm{PN}} \sim 2 \pi \frac{a_{1}^{5 / 2} c^{2}\left(1-e_{1}^{2}\right)}{3 k^{3}\left(m_{1}+m_{2}\right)^{3 / 2}}, \\
& t_{a_{2}^{-2}}^{1 \mathrm{PN}} \sim 2 \pi \frac{a_{2}^{5 / 2} c^{2}\left(1-e_{2}^{2}\right)}{3 k^{3}\left(m_{1}+m_{2}+m_{3}\right)^{3 / 2}}, \\
& t_{\mathrm{int}}^{1 \mathrm{PN}} \sim \frac{16}{9} \frac{a_{2}^{3} c^{2}\left(1-e_{2}^{2}\right)^{3 / 2}\left(m_{1}+m_{2}\right)^{3 / 2}}{\sqrt{a_{1}} e_{1} \sqrt{1-e_{1}^{2}} k^{3}\left(m_{1}^{2}+m_{1} m_{2}+m_{2}^{2}\right) m_{3}} .
\end{aligned}
$$

All of these timescales depend on the secularly varying, inner orbital eccentricity, which implies that we need to explore different eccentricity values in phase space. Equations (38)-(41) show that the Newtonian and 1PN timescales have a simple dependence on the inner and outer orbital eccentricity, on the mass ratio $q_{m}=m_{3} / m_{1}$, and on the SMAs.

If any of the above timescales is much smaller than all others, then the evolution of the triple is dominated by the corresponding term in the Hamiltonian. Next, we examine the three-body parameters where any two timescales are equal, which defines the region where the corresponding two terms are equally important. The corresponding regions are shown in Figure 4 in the $m_{2} \rightarrow 0$ limit.

Equating $t_{a_{1}^{-2}}^{1 \mathrm{PN}}$ to $t_{\text {quad }}^{\mathrm{N}}$ (Equations (37) and (39)) gives a relation between the SMAs which normalized to the gravitational radius of the inner and outer binaries, $R_{1}^{g}$ and $R_{3}^{g}$, as defined above, can be written as

$$
\left.\frac{a_{2}}{R_{3}^{g}}\right|_{\text {quad }=1 \mathrm{PN}\left(a_{1}^{-2}\right)} \sim\left(\frac{1}{3}\right)^{1 / 3}\left(\frac{a_{1}}{R_{1}^{g}}\right)^{4 / 3} \frac{1}{q_{m}^{2 / 3}} \frac{\left(1-e_{1}^{2}\right)^{1 / 3}}{\sqrt{1-e_{2}^{2}}} .
$$

This relation is shown by the blue hatched area bounded by solid blue lines in Figure 4 for $0 \leqslant e_{1} \leqslant 0.999$. A resonant-like $1 \mathrm{PN}$ excitation of eccentricity is possible in this region, as we will show in the next section. For much larger $a_{2}$ or smaller $a_{1}$, $t_{a_{1}^{-2}}^{1 \mathrm{PN}} \ll t_{\text {quad }}^{\mathrm{N}}$, and thus the Kozai-Lidov eccentricity excitations are suppressed by the $1 \mathrm{PN}$ effects.

Next, equating $t_{a_{2}^{-2}}^{1 \mathrm{PN}}$ to $t_{\text {quad }}^{\mathrm{N}}$ gives

$$
\left.\frac{a_{2}}{R_{3}^{g}}\right|_{\text {quad }=1 \mathrm{PN}\left(a_{2}^{-2}\right)} \sim 3\left(\frac{a_{1}}{R_{1}^{g}}\right)^{3} \frac{q_{m}}{\left(1+q_{m}\right)^{3}} \frac{1}{1-e_{2}^{2}} .
$$

This is shown by a blue short-long-dashed line in Figure 4 on the top and bottom panels for $e_{2}=0.5$ and 0.7 , respectively. For $a_{2} / R_{3}^{g}$ much larger than this value, the Kozai-Lidov oscillations are suppressed and the 1PN effects dominate.

Let us next compare $t_{\text {int }}^{1 \mathrm{PN}}$ and $t_{\mathrm{oct}}^{\mathrm{N}}$ by setting them equal to each other:

$$
\left.\frac{a_{2}}{R_{3}^{g}}\right|_{\text {oct }=1 \mathrm{PN}(\mathrm{int})} \sim \frac{32}{135 \pi}\left(\frac{a_{1}}{R_{1}^{g}}\right)^{2} \frac{1}{q_{m}} \frac{e_{1} e_{2}}{\left(1-e_{2}^{2}\right)\left(1-e_{1}^{2}\right)},
$$

shown by long-dashed brown lines in Figure 4. The 1PN effects are equally important as the Newtonian ones in the gray shaded area in Figure 4 for $0 \leqslant e_{1} \leqslant 0.999$. This is the regime in which the 1PN interaction term introduces qualitatively different behavior in the orbital motion (i.e., modulation) as shown in Figure 3. Outside the gray region in Figure 4, the interaction term is negligible. Note that comparing $t_{\text {int }}^{1 \mathrm{PN}}$ with $t_{\text {quad }}^{\mathrm{N}}$ leads to a vertical line in the phase diagram of Figure 4 . This is because both timescales have the same dependence on the outer SMA $\left(\sim a_{2}^{3}\right)$, resulting in $a_{1} / R_{1}<10$ (not shown). In Section 5, we explore the parameter space that also leads to excitations in the eccentricity (as shown in Figure 3).

Comparing $t_{a_{2}^{-2}}^{1 \text { PN }}$ to the octupole timescale gives usually a longer timescale than the quadrupole (short-dashed purple lines in Figure 4):

$$
\begin{aligned}
\left.\frac{a_{2}}{R_{3}^{g}}\right|_{\text {oct }=1 \mathrm{PN}\left(a_{2}^{-2}\right)} & \sim\left(\frac{4}{45}\right)^{1 / 3}\left(\frac{a_{1}}{R_{1}^{g}}\right)^{5 / 3} \\
& \times \frac{1}{q_{m}^{1 / 3}\left(1+q_{m}\right)^{5 / 3}} \frac{e_{2}^{2 / 3}}{\left(1-e_{2}^{2}\right)\left(1-e_{1}^{2}\right)^{1 / 3}}
\end{aligned}
$$

This relation also provides a range of parameters for different values of $e_{1}$ and $e_{2}$, but to avoid cluttering, we plot this timescale only for a circular inner binary $\left(e_{1}=0\right)$. The boundary shifts to larger $a_{2}$ in the eccentric case. In the top left panel of Figure 4 (the $q_{m}=0.01$ case), we show the range of Equation (45) for $e_{2} \in(0,0.999)$, since, in this case, this ratio is smaller than Equation (44).

Figure 4 also shows the regime of validity of the hierarchical triple approximation, where we choose $\epsilon>0.1$ as a rule of thumb for stability (dotted magenta line). For the $q_{m}=1$ case, this rule of thumb seems to agree with the Mardling 


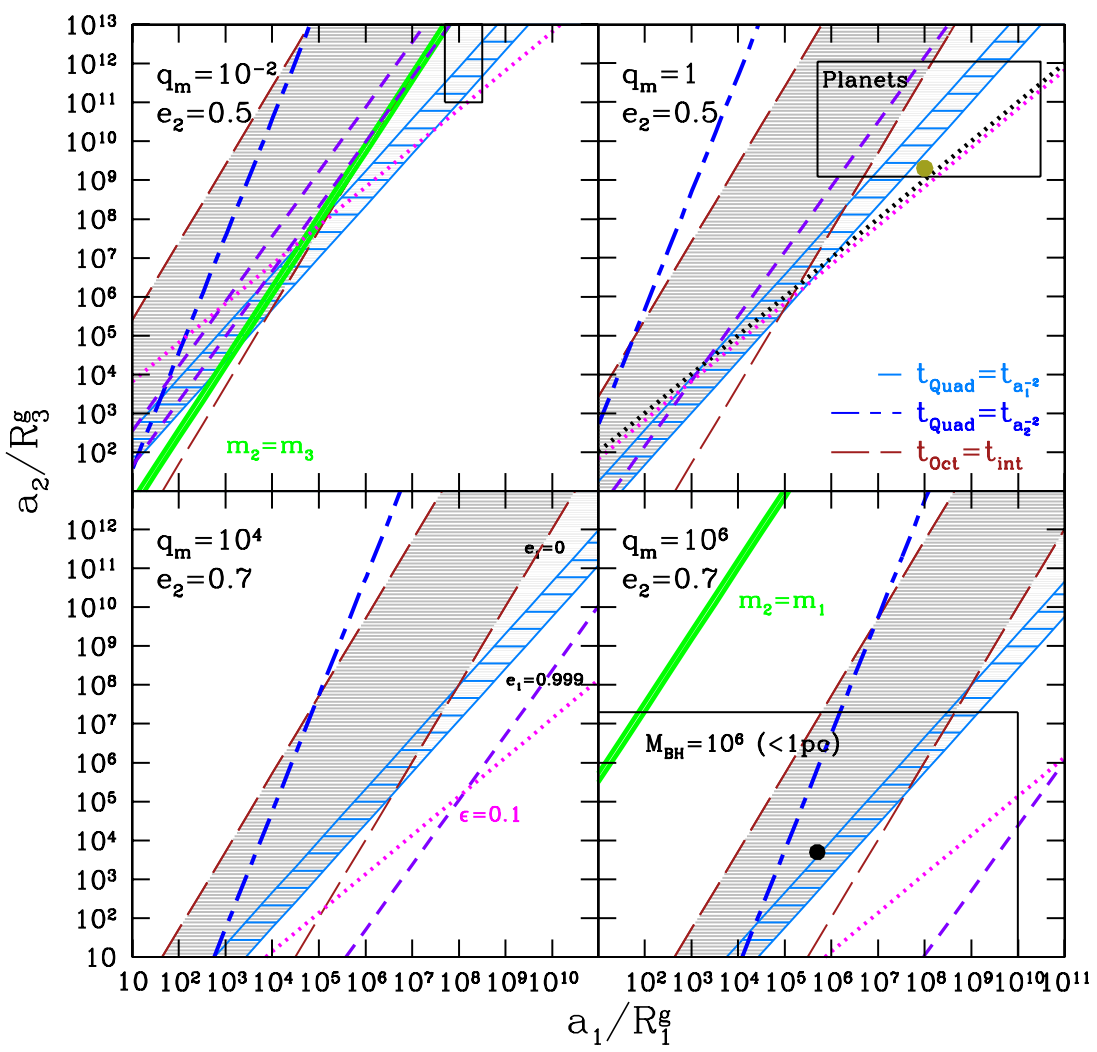

Figure 4. Regions of binary parameters where the various secular Newtonian and 1PN effects are expected to become significant. We show the SMAs where the timescales, corresponding to individual terms in the Hamiltonian, are equal to each other for different $a_{1} / R_{1}^{g}$ and $a_{2} / R_{3}^{g}$. We consider four mass ratios between the outer object and the inner binary $q_{m}=m_{3} / m_{1}=0.01,1,10^{4}$ and $10^{6}$ (see labels in each panel) and two different choices for outer orbital eccentricity $\left(e_{2}=0.5\right.$, top panels and $e_{2}=0.7$ lower panels), setting $m_{2} \rightarrow 0$ in all panels. We consider $t_{\text {quad }}^{\mathrm{N}}=t_{a_{1}^{-2}}^{1 \mathrm{PN}}$ (Equation (42); solid blue lines), $t_{\text {quad }}^{\mathrm{N}}=t_{a_{2}^{-2}}^{1 \mathrm{PN}}$ (Equation (43); thick long-short-dashed blue lines), $t_{\mathrm{oct}}^{\mathrm{N}}=t_{\mathrm{int}}^{1 \mathrm{PN}}$ (Equation (44); long-dashed brown lines) and $t_{\mathrm{oct}}^{\mathrm{N}}=t_{a_{2}^{-2}}^{1 \mathrm{PN}}$ (Equation (45); short-dashed purple lines). For the latter, we show $e_{1}=0$ (top purple line) in all panels and in the top left panel we also show the eccentric case $e_{1}=0.999$ (bottom purple line). The gray and blue shaded areas cover the range $0 \leqslant e_{1} \leqslant 0.999$ between the brown shaded lines and the blue solid lines, respectively. In the top left panel and bottom right, we also show a green band for which the timescale to shrink the inner orbit by a factor two, due to $\mathrm{GW}$ emission, is equal to the quadrupole timescale, which covers the range $0 \leqslant e_{1} \leqslant 0.999$. This line is generated by specifying $m_{1}=100 M_{\odot}$ and $m_{2}=m_{3}=1 M_{\odot}$ (top left panel) and $m_{2}=m_{1}=1 M_{\odot}$ (bottom right panel). We also show two stability criteria, $\epsilon=0.1$, dotted magenta lines and the Mardling \& Aarseth (2001) criterion (Equation (46); in the top right panel). The green dot in the right top panel represents (up to a factor three) the location in this phase space of the system considered in Figure 7, while the black dot in the bottom right panel represent the location of the example considered in Figure 3. The rectangle in the top left panel shows the parameter space considered in Figure 6. The black rectangles in the top and bottom right panels roughly represent the region in parameter space where planets in stellar binaries (top right) and stars in the galactic nuclei (bottom right) live.

(A color version of this figure is available in the online journal.)

\& Aarseth (2001) stability criterion, which defines a stable three-body system as one that obeys

$$
\frac{a_{2}}{a_{1}}>2.8\left(1+q_{m}\right)^{2 / 5} \frac{\left(1+e_{2}\right)^{2 / 5}}{\left(1-e_{2}\right)^{6 / 5}}\left(1-\frac{0.3 i_{\text {tot }}}{180^{\circ}}\right),
$$

where in the top right panel we considered this criterion with prograde $i_{\text {tot }}=0^{\circ}$. Note that retrograde motions are usually more stable (e.g., Innanen 1979, 1980; Morais \& Giuppone 2012).

Another consistency requirement for the 1PN Kozai-Lidov effects to operate is that gravitational radiation reaction does not change the SMA significantly over this timescale. We define $t_{\mathrm{GW} 1}$, the GW in-spiral timescale of the inner binary for the SMA to change by factor of two using Peters (1964) (see also Arun et al. 2009). Note that $t_{\mathrm{GW} 1} \rightarrow \infty$ when $m_{2} \rightarrow 0$, but the GW inspiral may become very important in the comparablemass limit and shut off the Kozai-Lidov effect. This is the case above the green bands in the top left and bottom right panels, which show the range of SMAs where $t_{\text {quad }}^{\mathrm{N}}=t_{\mathrm{GW} 1}$ for $m_{2}=m_{3}=1 M_{\odot}$ (top left panel) and $m_{2}=m_{1}=1 M_{\odot}$ (bottom right panel) for $0 \leqslant e_{1} \leqslant 0.999$. Note that there is a region below the green band where the 1PN approximation is insufficient, and where 2PN and higher PN order corrections need to be included; we leave this to future work. A similar regime, were the 1PN level of approximation is insufficient, was considered by Blaes et al. (2002) for which the evolution was mostly affected by GW emission and resonant eccentricity growth was not observed.

We conclude that 1PN effects may be important for a vast range of parameters as shown in Figure 4. Note however, that physical timescales are not shown there; this figure is independent of an overall dimensional scale (e.g., total mass or the scale of the SMAs). The physical timescale may be smaller or larger than the Hubble time depending on the actual system parameters considered.

\section{EXCITATION OF THE INNER ORBITAL ECCENTRICITY}

As stated above the 1PN effects may suppress the Newtonian Kozai-Lidov oscillations if the corresponding 1PN timescale is much smaller than the Newtonian quadrupole one. However, we identify two regimes where the combined secular 
Newtonian and 1PN effects produce interesting different behavior in three-body systems: (1) if the quadrupole order terms are comparable to or somewhat smaller than the 1PN ones, or (2) for comparable-mass inner binaries where the 1PN effects are subdominant relative to the Newtonian quadrupole terms but they are comparable to the Newtonian octupole timescale. We discuss these two regimes in detail below.

\subsection{Eccentricity Peak in the Restricted Three-body Problem for a Massive Perturber}

The standard lore says that GR effects (or 1PN effects in our case) suppress the eccentricity growth of the inner orbit in a three-body system (e.g., Blaes et al. 2002; Fabrycky \& Tremaine 2007). However, Ford et al. (2000b), studying the triple system PSR B1620-26, showed that the Newtonian octupole and the leading order 1PN corrections (i.e., Equation (35)) can lead to the excitation of the eccentricity of the inner orbit. We repeat and extend the investigations of that study for a broader range of three-body systems and examine (1) whether the new 1PN terms derived in Section 2 give rise to different behavior; and (2) whether Newtonian octupole terms can significantly change the evolution of the three-body system in the presence of the 1PN terms by producing flips of the inner orbit and eccentricities close to unity (Naoz et al. 2011, 2013). We begin by exploring systems in which $m_{3} \gg m_{1}\left(q_{m} \gg 1\right)$, and investigate the opposite limit in the next subsection.

Let us systematically examine the parameters where the competition between the secular Newtonian Hamiltonian and 1PN corrections lead to the excitation of the inner orbital eccentricity as opposed to a suppression. We do this by preforming a large number of simulations starting from a nearly zero eccentricity for the inner binary and varying the following dimensionless parameter:

$$
\mathcal{R}=\left.\frac{t_{a_{1}^{-2}}^{1 \mathrm{PN}}}{t_{\text {quad }}^{\mathrm{N}}}\right|_{e_{1}=0}=\frac{1}{3} \frac{\left(a_{1} / R_{1}^{g}\right)^{4}}{\left(a_{2} / R_{3}^{g}\right)^{3}} \frac{1}{q_{m}^{2}\left(1-e_{2}^{2}\right)^{3 / 2}},
$$

where we have substituted Equations (37) and (39) with $e_{1}=0$. The quantity in Equation (47) compares the timescales of the leading order 1PN and secular Newtonian effects. We find that a resonant-like eccentricity excitation can take place around $\mathcal{R}=1$ for zero initial eccentricity as shown below. Note, however, that another important ingredient to this resonant-like behavior is the Newtonian octupole term, which is most obvious in simulations with a low initial mutual inclination, for which the quadrupole approximation is subdominant (see also Section 5.2 below and Ford et al. 2000b). In other words, neglecting the contribution of the octupole-level of approximation, one would miss entirely the resonant behavior.

Figure 5 shows the maximum eccentricity achieved during the course of the evolution of the system studied, after 1000 quadrupole cycles ${ }^{10}$ as a function of $\mathcal{R}$. We also examined the effects of various $1 \mathrm{PN}$ terms by repeating the calculations using $\overline{\mathcal{H}}_{\mathrm{N}}+\overline{\mathcal{H}}_{a_{1}^{-2}}^{1 \mathrm{PN}}$ (blue triangles), then adding $\overline{\mathcal{H}}_{a_{2}^{-2}}^{1 \mathrm{PN}}$ (green crosses), and finally including $\overline{\mathcal{H}}_{\text {int }}^{1 \mathrm{PN}}$ (red circles). The fiducial

\footnotetext{
${ }^{10}$ Each point in this figure corresponds to a separate, high-resolution three-body evolution with 1PN effects, each of which takes approximately three days to complete per computer core. We also preformed convergence tests using longer integration times at high resolutions, and found that for this type of systems at least 1000 quadrupole cycles are needed for convergence, over the parameter range considered in the figure.
}

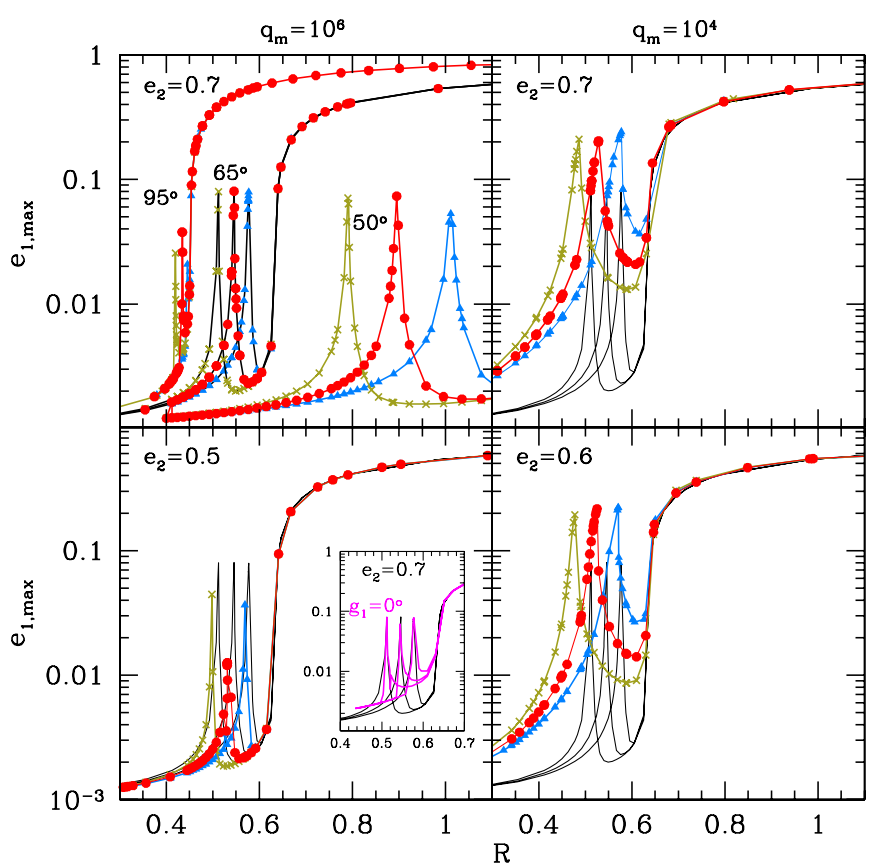

Figure 5. The maximum eccentricity in a triple system in the test-particle approximation for different mass ratios $\left(q_{m}=10^{6}\right.$, left-hand panels, and $q_{m}=10^{4}$, right-hand panels) as a function of the relative timescales of the $1 \mathrm{PN}$ and secular Newtonian quadrupole effects $(\mathcal{R}$; see Equation (47)). We consider the 1PN evolution including terms only up to $\mathcal{O}\left(a_{1}^{-2}\right), \mathcal{O}\left(a_{2}^{-2}\right)$ and the interaction term (blue triangles, green crosses, and red squares, respectively). We show three different initial outer orbital eccentricities: $e_{2}=0.7$ (top panels) $e_{2}=0.6$ and 0.5 , bottom right and left respectively. In all these examples, we set $m_{1}=1 M_{\odot}, m_{2}=0.001 M_{\odot}, m_{3}=q_{m} m_{1}$ and we vary both the inner and outer SMAs to match the different values of $\mathcal{R}$, see Equation (47). The system is initialized with $e_{1}=0.001, g_{2}=0^{\circ}$ and $g_{1}=240^{\circ}$ (inset shows the results for initializing $g_{1}=0^{\circ}$ and $240^{\circ}$ ). In all panels we set the initial mutual inclination to $65^{\circ}$. In the top left panel, we also set the initial inclination to $95^{\circ}$ (left set of lines) and $50^{\circ}$ (right set of lines). We compare the fiducial example $\left(q_{m}=10^{6}, i_{\text {tot }}=65^{\circ}, e_{2}=0.7\right)$, black lines in each panel, to systems with a different mass ratio $\left(q_{m}=10^{4}\right)$, right hand panels), and different eccentricities, see labels top right panel. Observe the emergence of resonant-like eccentricity excitations.

(A color version of this figure is available in the online journal.)

example chosen (black lines in all panels) has $m_{1}=1 M_{\odot}$, $m_{2}=0.001 M_{\odot}$, and $m_{3}=10^{6} M_{\odot}$ (i.e., $q_{m}=10^{6}$ ) with initial conditions $e_{1}=0.001, e_{2}=0.7, i_{\text {tot }}=65^{\circ}, g_{1}=240^{\circ}$ and $g_{2}=0^{\circ}$. To generate Figure 5 , we used different runs and changed both $a_{1}$ and $a_{2}$. For a choice of mass ratio and initial mutual inclination the parameter $\mathcal{R}$ collapses the different runs with different SMAs into one curve. Note that changing the initial value of the argument of periapsis does not change the location of the resonance, but it does change its width, as shown in the inset of Figure 5, where we compare the fiducial example with an initial $g_{1}=240^{\circ}$ to an initial $g_{1}=0$. The different panels show that the amplitude and width of the resonance are different for different $q_{m}$ and $e_{2}$, as they depend on the Newtonian octupole terms. The top left panel shows that different initial inclinations change both the location of the resonance and its width (as a function of $\mathcal{R}$ ), i.e., low initial inclination leads to broad resonances. The time evolution of the systems with parameters on the peak of the resonance of the $i_{\text {tot }}=95^{\circ}$ and $65^{\circ}$ cases are shown in Figure 3.

To the left of the resonance peak, the maximum eccentricity converges asymptotically to the initial inner eccentricity for $\mathcal{R} \ll 1$ (i.e., in this case the eccentricity is not excited). This was chosen to be $e_{1}=10^{-3}$ in Figure 5. Decreasing the initial 
eccentricity (not shown) changes the figure at $\mathcal{R} \ll 1$, but it does not change the amplitude and location of the resonance and the behavior at larger $\mathcal{R}$. To the right of the resonance $(\mathcal{R} \gtrsim 1)$, the maximum eccentricity starts to increase when the eccentric Kozai-Lidov mechanism begins to dominate. For $q_{m} \gg 1$ and $\mathcal{R} \gg 1$, we find asymptotically $e_{1} \rightarrow 1$ (see Section 5.2 for small $q_{m}$ ).

A binary that slowly shrinks due to $\mathrm{GW}$ emission, slowly changes $\mathcal{R}$ and may sweep across the resonant regions of eccentricity excitation shown in Figure 5. Thus, a resonant 1PN eccentricity increase may take place in many inspiraling systems some time during their evolution. Whether or not this occurs depends on the masses and SMAs; a detailed analysis is left to future work.

\subsection{The Case of PSR B1620-26}

Although the above discussion of the resonant behavior assumed a test particle $\left(m_{2} \sim 0\right)$, general mass-ratio triples also exhibit a similar effect (as was first considered by Ford et al. $2000 \mathrm{~b}$ ), provided $t_{a_{1}^{-2}}^{1 \mathrm{PN}}$ is shorter then $t_{\text {quad }}^{\mathrm{N}}$ (see Equation (39)). To find $\mathcal{R}$ in the general case, we can simply set

$$
q_{m} \equiv m_{3} /\left(m_{1}+m_{2}\right)
$$

in Equation (47). Ford et al. (2000b) observed a resonantlike eccentricity increase while studying the triple system PSR B1620-26, which is located near the core of the globular cluster M4. They showed that this resonant behavior may explain the unusually large eccentricity of the inner binary, which contains a millisecond radio pulsar of $m_{1}=1.4 M_{\odot}$ and a companion of $m_{2}=0.3 M_{\odot}($ McKenna \& Lyne 1988).

For completeness, we repeat and extend the calculation of Ford et al. (2000b) by fixing the inner orbit's SMA, changing the outer orbit's SMA, and including all 1PN terms. We choose two different values for the inner binary to explore the sensitivity to these parameters. First, following Ford et al. (2000a), we consider $a_{1} / R_{1}=5.6 \times 10^{7}$ (i.e., $a_{1}=0.77 \mathrm{AU}$ ). Additionally, we consider $a_{1} / R_{1}=3.6 \times 10^{8}$ (i.e., $a_{1}=5 \mathrm{AU}$ ). We adopt parameters for the outer perturber from Ford et al. (2000a): $m_{3}=0.01 M_{\odot}$ and $e_{2}=0.45$. We initialize the system with $g_{1}=g_{2}=0^{\circ}, e_{1}=10^{-4}$, and we also choose two different initial inclinations, $i_{\text {tot }}=65^{\circ}$ and $i_{\text {tot }}=0^{\circ}$. A mutual inclination of $i_{\text {tot }}=0^{\circ}$ highlights that the perturbations of the outer orbit affect the inner orbit due to the Newtonian octupole term, even far from the nominal Kozai-Lidov regime. In this configuration, the $\overline{\mathcal{H}}_{a_{1}^{-2}}^{1 \mathrm{PN}}$ term is the most significant $1 \mathrm{PN}$ effect, as shown in Figure 4 (see the rectangle in the top left panel).

The left panel of Figure 6 shows the inner orbit's maximum eccentricity as a function of the outer orbit's SMA (or equivalently the $\mathcal{R}$ value for $a_{1}=0.77 \mathrm{AU}$ ). This figure confirms the resonant-like increase in eccentricity found in Ford et al. (2000b, their Figure 14). This figure also shows that the resonant-like eccentricity increase is present for a large range of $a_{2}$ values, even for systems where the excitation of the eccentricity due to the Newtonian octupole term is somewhat suppressed, due to comparable masses for the inner orbit (e.g., Naoz et al. 2013). Although changes in the outer orbit's eccentricity (see the right panels of Figure 6) do not change the location of the resonant peaks, their amplitude does change for large inclinations. The $a_{1}=5$ AU case has irregular behavior and results in a higher inner and outer orbital eccentricity. Note that $\mathcal{H}_{a_{1}^{-2}}^{1 \mathrm{PN}}$ term is the dominant one here and the other 1PN terms are negligible in
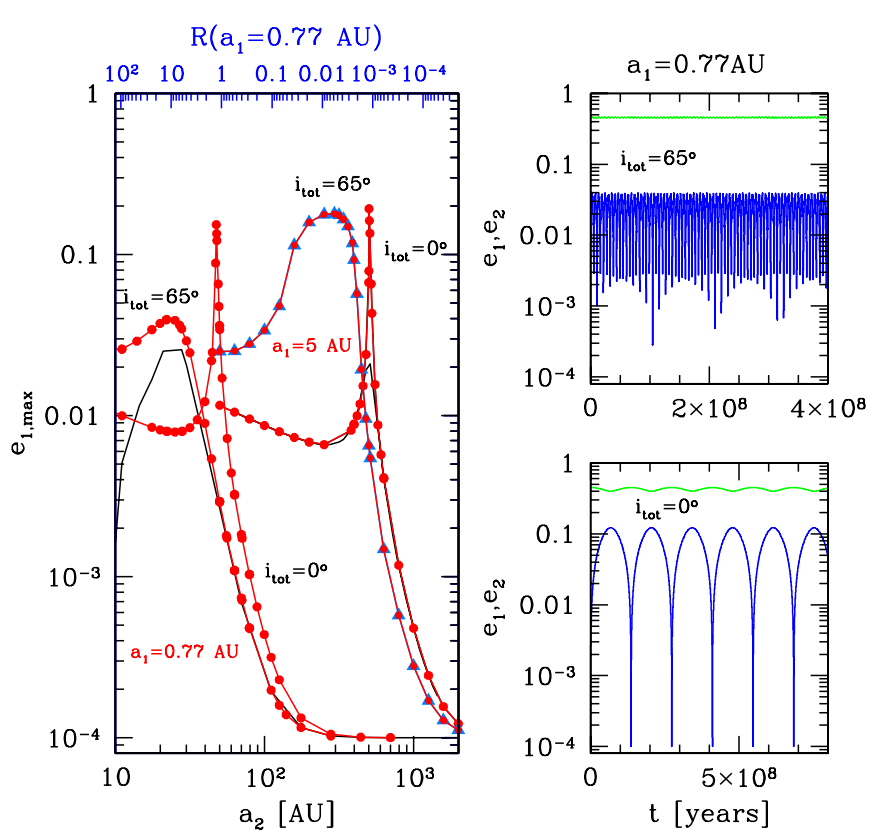

Figure 6. The maximum eccentricity as a function of the outer orbit's SMA (left panel) in a triple for the case of PSR B1620-26. The inner binary is a millisecond pulsar of mass $1.4 M_{\odot}$ with a companion of $m_{2}=0.3 M_{\odot}$, and the outer body has mass $m_{3}=0.01 M_{\odot}$. The inner orbit has $a_{1}=5 \mathrm{AU}$ and $0.77 \mathrm{AU}$, in two different sets of simulations (see labels). The initial eccentricities are $e_{1}=10^{-4}$ and $e_{2}=0.45$ and the initial relative inclination $i_{\text {tot }}=65^{\circ}$ and $i_{\text {tot }}=0^{\circ}$. The argument of pericenter of the inner and outer orbits are initially set to zero. The top axis show the value of $\mathcal{R}$ for the case of $a_{1}=0.77 \mathrm{AU}$; see Equation (47). Note that the $i_{\text {tot }}=0^{\circ}$ resonance happens when $\mathcal{R} \sim 1$. The thin black lines show the results of integrating the system over a single Kozai-Lidov cycle. The left thin black line is for the case of $a_{1}=0.77 \mathrm{AU}$ and $i_{\text {tot }}=65^{\circ}$, while the right one is for $a_{1}=5 \mathrm{AU}$ and $i_{\text {tot }}=0^{\circ}$. Although we include all $1 \mathrm{PN}$ terms described in previous sections, the curves corresponding to the lower order ones exactly overlap the curves including the interaction term (i.e., the leading 1PN term is the most dominant in the evolution of the system). In the right panels, we show the time evolution of the eccentricity of the inner (blue line) and outer binary (green line). Here we set $a_{1}=0.77 \mathrm{AU} i_{\text {tot }}=0^{\circ}, a_{2}=48 \mathrm{AU}$ in the bottom and $i_{\text {tot }}=65^{\circ}, a_{2}=22 \mathrm{AU}$ in the top panel, respectively. In this case, the resonant eccentricity excitation due to the 1PN terms reaches higher values than the one achieved by the eccentric Kozai-Lidov mechanism for small (large) $a_{2}(\mathcal{R})$.

(A color version of this figure is available in the online journal.)

this configuration, as can be see from the black rectangle in the top left panel in Figure 4.

The right panels of Figure 6 show the time evolution of the inner and outer orbital eccentricity for the $a_{1}=0.77$ AU case with $i_{\text {tot }}=0^{\circ}$ and $a_{2} / R_{3}=4.9 \times 10^{11}\left(a_{2}=48 \mathrm{AU}\right.$, bottom panel) and $i_{\text {tot }}=65^{\circ}$ and $a_{2} / R_{3}=2.2 \times 10^{11}\left(a_{2}=22 \mathrm{AU}\right.$, top panel). Unlike the systems considered in Figure 5, the outer orbit's eccentricity oscillates slightly (see right-hand panels in Figure 6). Furthermore, the eccentricity peak is even larger than the eccentricity reached for $\mathcal{R} \gtrsim 1$, which shows that the 1 PN terms further increase the inner orbit's eccentricity above the excitation induced by the Newtonian eccentric Kozai-Lidov mechanism. This was not the case in Figure 5 primarily because there $q_{m} \gg 1$.

Although we integrated the system for up to 1000 Kozai-Lidov cycles, the eccentric Kozai-Lidov process did not seem to induce chaotic behavior in this configuration (since $\left.m_{3}<m_{1}+m_{2}\right)$. Numerical convergence was reached already after a few hundred Kozai-Lidov cycles of evolution (in thin black lines we show the results of integrating the system up to 1 Kozai-Lidov cycle). Note that this system was integrated both using the calculation presented here and using direct 
three-body in Ford et al. (2000b, Figure 15); here we find perfect agreement with their results.

The top right panel of Figure 6 shows that integrating over only one Kozai-Lidov cycle, for the $i_{\text {tot }}=65^{\circ}$ case, misses the long timescale oscillation, since the largest eccentricity is reached only after several Kozai timescales. This explains why our eccentricity peaks are slightly higher than those of Ford et al. (2000b), as we see for the $i_{\text {tot }}=65^{\circ}$ case in the left panel. This also explains the somewhat larger $e_{1, \max }$ values we found compared to Ford et al. (2000b) in the regime where the $1 \mathrm{PN}$ effects are subdominant and the eccentricity of the inner orbit increases due to the eccentric Kozai-Lidov mechanism. Note, however, that the lifetime of this system in the core of the globular M4 is about one Kozai-Lidov cycle, which explains why Ford et al. (2000b) did not bother to evolve over many Kozai-Lidov cycles.

\subsection{Orbital Flips and Eccentricity Excitation for Comparable-mass Inner Binary}

If the inner binary consists of comparable mass-objects, the Newtonian octupole term is suppressed (see Equation (21)). Recently, Sharpee \& Thompson (2013) considered the evolution of triple systems with comparable masses and showed that the eccentric Kozai-Lidov evolution can be triggered if one of the stars in the inner binary loses mass. We show here that the eccentric Kozai-Lidov evolution can also be triggered without mass loss, but accounting for 1PN effects, as shown in Figure 7 . For this figure, we set $m_{1}=10 M_{\odot}, m_{3}=30 M_{\odot}$, $a_{1} / R_{1}^{g}=1 \times 10^{8}$ (corresponding to $10 \mathrm{AU}$ ), and $a_{2} / R_{3}^{g}=$ $1.7 \times 10^{9}$ (corresponding to $502 \mathrm{AU}$ ). The initial eccentricities were $e_{1}=0.001$ and $e_{2}=0.7$ and the initial relative inclination $i_{\text {tot }}=94^{\circ}$. The argument of pericenter of the inner and outer orbits was initially set to $240^{\circ}$ and zero, respectively. The two panels in this figure differ in the choice of $m_{2}$, i.e., in the left panel $m_{2}=0.001$ and in the right panel $m_{2}=8 M_{\odot}$. While a test-particle evolution is relatively insensitive to the 1PN terms in this case, comparable mass systems present qualitatively different behavior. In particular, while the Newtonian eccentric Kozai-Lidov effect is suppressed for comparable masses, 1PN effects can trigger it. A possible reason for the qualitative difference is that changing $m_{2}$ from zero to $8 M_{\odot}$ resulted in a configuration for which the leading order 1PN timescale is closer to (but still slightly longer than) the octupole timescale. In the test particle case, the octupole timescale is two orders of magnitude shorter than the shortest 1PN timescale.

The excitation of the eccentricity depends on the importance of the Newtonian octupole term. Without 1PN effects, comparable mass triples result in $\epsilon_{M} \rightarrow 0$ (Equation (21)), which suppresses the eccentricity excitations and the flipping of the inner orbits. This can be seen in Figure 8 (black and gray lines) for two examples, where we consider $\Delta m=\left|m_{1}-m_{2}\right|=2 M_{\odot}$, (black lines) and $\Delta m=0.2 M_{\odot}$ (gray lines). For both of these, $m_{3}=3 m_{1}$ and the two systems were initialized with $e_{2}=0.7$, $e_{1}=0.001$ and $i_{\text {tot }}=94^{\circ}$. However, we find that although 1PN effects are small during a single orbit, they can be significant on much longer timescales, and can lead to significant eccentricity growth and orbital-flips. ${ }^{11}$ The top panel shows that for $\epsilon_{M} \geqslant 0.002$ a flip is triggered due to the $1 \mathrm{PN}$ terms. In other words, for $\epsilon_{M} \geqslant 0.002$ the colored curves deviate (including

\footnotetext{
11 The choice of argument of periapsis does not change the outcome; the first example was initialized with $g_{1}=240^{\circ}$ while the second had $g_{1}=0^{\circ}$, both had $g_{2}=0^{\circ}$.
}
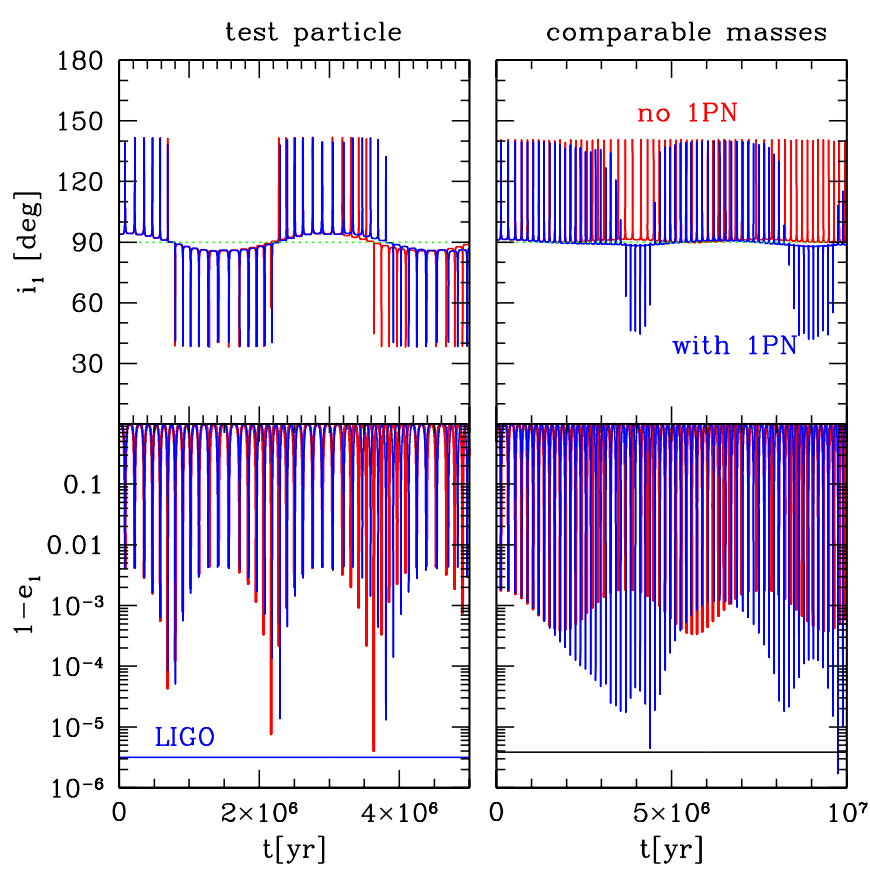

Figure 7. Eccentricity excitation and orbital flips for the Newtonian octupole and the 1PN approximations (up to the interaction term; see text for details) as a function of time. We compare the test-particle case $\left(m_{2} \rightarrow 0\right.$, left panels) to a comparable mass case $\left(m_{2}=8 M_{\odot}\right.$, right panels) in the inner binary with $m_{1}=10 M_{\odot}$, always with an outer object of mass $m_{3}=30 M_{\odot}$. The separation of the inner orbit is $a_{1} / R_{1}^{g}=1 \times 10^{8}$ (corresponding to $10 \mathrm{AU}$ and orbital period $P_{1} \sim 10 \mathrm{yr}$ ), and the outer orbit's separation is $a_{2} / R_{3}^{g}=1.69 \times 10^{9}$ (corresponding to $502 \mathrm{AU}$ and $P_{2}=2.8 \times 10^{3} \mathrm{yr}$ ). The initial eccentricities are $e_{1}=0.001$ and $e_{2}=0.7$ and the initial relative inclination $i_{\text {tot }}=94^{\circ}$. The arguments of pericenter of the inner and outer orbits are initially set to $240^{\circ}$ and zero respectively. For these examples $\mathcal{R} \gg 1$. We show with red lines evolutions without $1 \mathrm{PN}$ corrections (curves including only the lower order $1 \mathrm{PN}$ terms simply overlap this). We also show the minimum eccentricity corresponding to the detectable LIGO frequency range (horizontal lines in the bottom panels). The 1PN corrections help to further increase the eccentricity and lead to orbital flips for the inner binary for comparable masses.

(A color version of this figure is available in the online journal.)

1PN effects) from the black and gray ones (Newtonian effects only). For larger $\epsilon_{M}$, where the eccentric Kozai-Lidov mechanism is triggered, the maximum eccentricity can be very close to unity, and thus due to the chaotic nature of the system, the maximum eccentricity shown should be considered as a lower limit. For $\epsilon_{M} \gg 0.005$ we could not reach convergence after 1000 quadrupole cycles, since the eccentricity is very close to unity (see J. Teyssandier et al., in preparation).

In the above examples, a requirement for eccentricity excitation is that the $1 \mathrm{PN}$ timescale, $t_{a_{1}^{-2}}^{1 \mathrm{PN}}$, be shorter than or comparable to the octupole timescale $t_{\mathrm{oct}}^{\mathrm{N}}$, (see Equation (38)), i.e., $t_{\text {quad }}^{\mathrm{N}} \lesssim t_{a_{1}^{-2}}^{1 \mathrm{PN}} \sim t_{\mathrm{oct}}^{\mathrm{N}}$. A possible explanation for the excitation of the eccentricity in these cases is the following. Neglecting $1 \mathrm{PN}$ effects, comparable masses in the inner binary suppress the Newtonian octupole effects and the outer potential is effectively quadrupolar. However, GR precession of the inner orbit breaks this symmetry. As long as GR precession occurs on a timescale comparable to (or slightly smaller than) the octupole one, the eccentric Kozai-Lidov mechanism is then triggered.

Let us now discuss the implications of these finding for direct GW detections using Earth-based instruments like LIGO and VIRGO. The characteristic frequency of the GW signal is $f_{p}=v_{p} / r_{p}$, where $v_{p}$ and $r_{p}$ are the orbital velocity and 


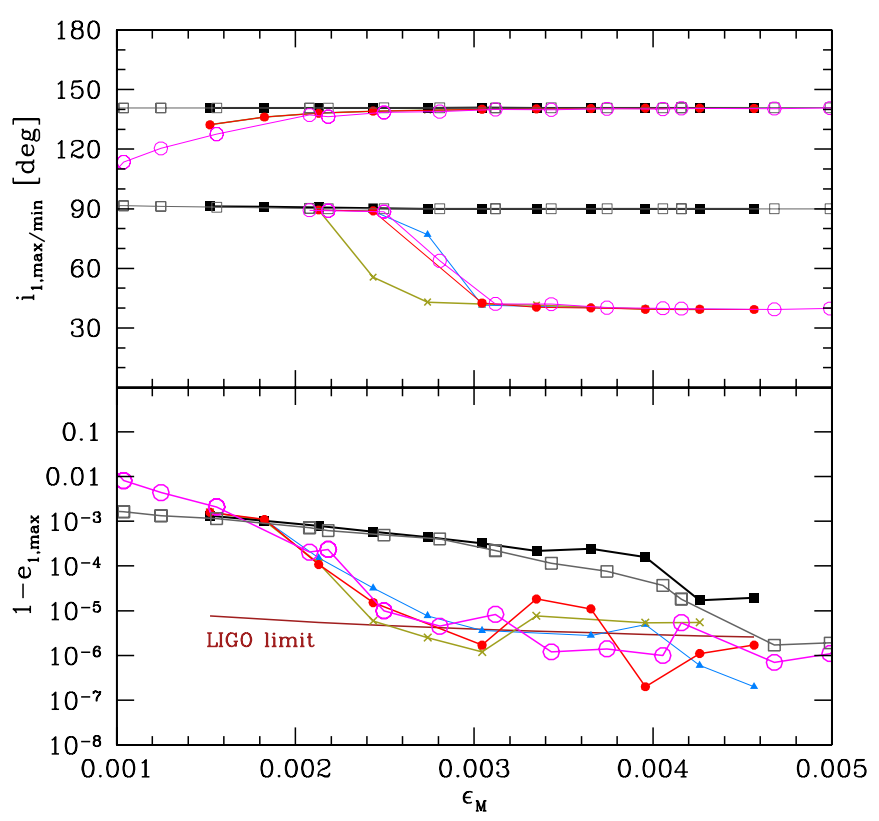

Figure 8. Excitation of the eccentricity and inclination due to $1 \mathrm{PN}$ effects for Kozai-Lidov timescales much shorter than the lowest 1PN timescales (as parameterized through $\epsilon_{M}$; see Equation (21)). We examine the maximum eccentricity reached, and plot $1-e_{1, \max }$ (bottom panel), and the maximum and minimum inclination reached in the runs (top panel). We show two examples. The first system is initialized with $m_{1}=10 M_{\odot}, m_{2}=8 M_{\odot}, m_{3}=30 M_{\odot}$, and $e_{2}=0.7$. We initialize the system with $e_{1}=0.001, g_{1}=240^{\circ}, g_{2}=0$, and $i_{\text {tot }}=94^{\circ}$. We vary both the inner and outer SMAs to match the different values of $\epsilon_{M}$ depicted in the figure. We consider the Newtonian approximation (filled black squares) and the three 1PN level of approximations, $\mathcal{O}\left(a_{1}^{-2}\right), \mathcal{O}\left(a_{2}^{-2}\right)$ and the interaction term (filled blue triangles, green crosses, and filled red squares, respectively). In the second system, we set $m_{1}=1 M_{\odot}, m_{2}=1.2 M_{\odot}$ $m_{3}=3 M_{\odot}$ and $e_{2}=0.7$. We initialize the system with $e_{1}=0.001, g_{1}=0^{\circ}$, $g_{2}=0$ and $i_{\text {tot }}=94^{\circ}$. We vary both the inner and outer SMAs to match the different values of $\epsilon_{M}$ depicted in the figure. For this example, we consider the Newtonian approximation (empty gray squares) and up to the interaction level of the 1PN approximation (empty magenta circles). We also show the detectable LIGO frequency limit for the first example, where we set $a_{2}=501 \mathrm{AU}$ and varied $a_{1}$ between $5 \mathrm{AU}$ and $15 \mathrm{AU}$ (solid brown line).

(A color version of this figure is available in the online journal.)

radius at pericenter (Peters 1964). Thus, $f_{p}=2 \pi(1+e)^{1 / 2}(1-$ $e)^{-3 / 2} P^{-1}$, where $P$ is the orbital period. We assume that the GW signal is in the detectable frequency band if $f_{p}>5 \mathrm{~Hz}$. For a comparable-mass inner binary, the Newtonian eccentric Kozai-Lidov mechanism is suppressed ${ }^{12}$ and the eccentricity remains smaller than in the test particle case. The GW frequency emitted by a circular binary with an orbital period larger than a second is too small for a LIGO detection. However, the 1PN eccentricity excitations discussed in this paper lead to a much larger $f_{p}$ and might lead to GWs in the LIGO band. In particular, Figures 7 and 8 show examples where $1-e_{1}$ can be as small as $10^{-5}$ to $10^{-6}$ for a comparable-mass inner binary. These sources enter the LIGO GW frequency band if their orbital period is less than 1-60 yr. However, note that this estimate is oversimplified because it neglects the backreaction of GW emission on the evolution. The latter strongly reduces the SMA of the binaries during close approaches, and may lead to an eccentric inspiral and merger within a Kozai-Lidov period. The GW inspiral may deliver the binaries to the LIGO frequency band even if the signal is outside the LIGO band during the Kozai-Lidov oscillations. If the event rate of these sources is sufficiently large within the

12 Although suppressed, note that the eccentricity can still reach 0.999 in this case.
LIGO detection range, these sources could constitute a distinct population for LIGO (Wen 2003; Antonini \& Perets 2012).

\section{DISCUSSION}

The Kozai-Lidov mechanism (Kozai 1962; Lidov 1962; see below), has been shown to play an important role for highly inclined hierarchical triples, from planetary systems to stellar size and/or massive compact objects (e.g., Naoz et al. 2013, and references therein). For an eccentric outer perturber, the eccentricity of the inner orbit can reach values extremely close to unity, and the inclination can flip from prograde to retrograde (Naoz et al. 2011, 2013). The quadrupole Kozai-Lidov oscillations between the eccentricity and inclination still persist at octupole order, but they are further modulated on long timescales.

We have here studied how the Kozai-Lidov mechanism is affected by 1PN corrections to the three-body Hamiltonian, focusing on secular and hierarchical three-body systems. We expanded the 1PN Hamiltonian in the ratio of SMAs $(\alpha)$ to third order beyond leading, i.e., the leading-order terms in the 1PN Hamiltonian perturbation scale here as $a_{1}^{-2}$ and we carried out an expansion up to relative $\mathcal{O}\left(\alpha^{3}\right)$. We also averaged over the orbital timescale of the inner and outer binary to investigate the long-term secular evolution of the system (Section 2). We examined the effects of the different $1 \mathrm{PN}$ terms in this expansion: 1PN precession of the inner orbit due to $\overline{\mathcal{H}}_{a_{1}^{-2}}^{1 \mathrm{PN}}$ (Equation (28)); 1PN precession of the outer orbit due to $\overline{\mathcal{H}}_{a_{2}^{-2}}^{1 \mathrm{PN}}$ (Equation (30)); and a new 1PN interaction term between the two orbits, $\overline{\mathcal{H}}_{\text {int }}^{1 \mathrm{PN}}$ (Equation (31)), which introduces a new inclination and eccentricity dependent modulation (e.g., Figure 3 ).

We compared the different timescales associated with the secular Newtonian and different 1PN terms (see Figure 4). If the timescales associated with the 1PN effects are much shorter than the timescales associated with the eccentric Kozai-Lidov mechanism, i.e., the secular Newtonian timescales, the growth of the eccentricity in the inner orbit tends to be suppressed. We confirm that the excitation of the eccentricity is indeed suppressed for systems where the Kozai-Lidov timescale is many orders of magnitude longer than the 1PN timescales. However, if the timescales of the 1PN effects are comparable to the secular Newtonian ones (see Figure 4), we found two interesting regimes that present qualitatively different behavior.

The first regime is where the 1PN timescales are comparable but slightly shorter than the Newtonian Kozai-Lidov timescale. Ford et al. (2000b), studying the PSR B1620-26 triple system, noted that the inner eccentricity may be greatly increased around some critical value of the outer SMA, due to the $\overline{\mathcal{H}}_{a_{1}^{-2}}^{1 \mathrm{PN}}$ term and the octupole term. We extended this calculation by including all averaged 1PN terms up to $\mathcal{O}\left(\alpha^{3}\right)$ and the Newtonian octupole term (Naoz et al. 2013), as well as exploring a wide region of phase space. We confirmed Ford et al. (2000b) result and found a resonant-like behavior, where the inner orbital eccentricity is greatly increased compared to the Newtonian case. This behavior exists also when including all averaged 1PN terms and for a wide range of mass ratios and orbital parameters. We parameterized the location of the resonant peak in terms of the SMAs by defining a parameter, $\mathcal{R}$ in Equation (47), as the ratio of the leading-order 1PN and secular Newtonian terms. This parameter depends on the ratio of the mass of the outer perturber to the total mass of the inner binary. The presence of the octupole term is important for the resonant $1 \mathrm{PN}$ eccentricity 
excitation, which is most apparent in the examples with a small mutual inclination. For systems where either the inner or the outer binary shrinks, for example due to $\mathrm{GW}$ radiation reaction, the triple may pass through this three-body 1PN resonance. The amplitude and location of the resonance changes due to $1 \mathrm{PN}$ terms as a function of $\mathcal{R}$. We found that lower mutual inclinations in the prograde regime cause a wider peak (in terms of $\mathcal{R}$ ), while a less massive outer body tends to produce wider and higher amplitude peaks. A detailed investigation of the properties of the resonance is beyond the scope of this paper, but could be the subject of future investigations.

It is important to note that the outer orbit precession and the interaction term affect the overall time evolution (see Figure 3). Since these terms are a result of the expansion of the threebody $1 \mathrm{PN}$ Hamiltonian in $\alpha$, it is not surprising that the different terms affect the location of the resonant like behavior (e.g., Figure 5). It is interesting, however, that they produce a qualitatively different time evolution of the system (e.g., bottom panels of Figure 3 ). This suggests that a system evolved under GR effects in the presence of a third body has richness to it that should be examined in more detail. This is the subject of future investigation in the framework of direct three-body integration.

The second regime that exhibits qualitatively different behavior from that obtained with a quadrupole Newtonian Kozai-Lidov treatment is when the quadrupolar secular Newtonian timescales are shorter than the 1PN ones and when the inner binary has comparable mass components. The eccentric Kozai-Lidov mechanism, neglecting 1PN effects, is suppressed when $m_{1} \rightarrow m_{2}$, since the outer orbit's potential is effectively quadrupolar. As we showed in this paper, 1PN effects can break symmetry and excite eccentricity, triggering the eccentric Kozai-Lidov mechanism. As long as 1PN precession occurs on a comparable timescale (or lower) than the Newtonian octupole precession, i.e., $t_{\text {quad }}^{\mathrm{N}} \lesssim t_{a_{1}^{-2}}^{1 \mathrm{PN}} \sim t_{\mathrm{oct}}^{\mathrm{N}}$, the eccentric Kozai-Lidov mechanism will be triggered.

Eccentricity excitations are particularly interesting in the context of possible GW detections (Wen 2003; Brown \& Zimmerman 2010; Armitage \& Natarajan 2005; Sesana 2010).
If such excitations were not present, the frequency of the GWs emitted by the inner binary would be typically too low for detection with LIGO (see however O'Leary et al. 2009; Kocsis \& Levin 2012, for eccentric binaries which form in the LIGO band). However, if eccentricity is secularly excited through a three-body interaction, the frequency of the GWs is also increased during pericenter passage, thus bringing the signals into the detector's sensitivity band. Such large eccentricities would then lead to GW-driven inspiral and the eventual merger of binaries. Whether such eccentric signals can be detected or not will depend on how close such sources are to Earth. But if detections are made with sufficiently high signal-to-noise ratio, then GWs could be used to measure the eccentricity of the inner binary, and thus, distinguish between different source populations.

We thank Alessandra Buonanno, Fred Rasio, and Gongjie $\mathrm{Li}$ for useful discussions, and we also thank Cole Miller for carefully reading the first draft of the paper and sending useful comments. We thank our anonymous referee for useful remarks. We thank Yoram Lithwick for the use of his allocation time on the computer cluster Quest. This research was supported in part through the computational resources and staff contributions provided by Information Technology at Northwestern University as part of its shared cluster program, Quest. S.N. acknowledge partial supported by NASA through an Einstein Post-doctoral Fellowship awarded by the Chandra X-Ray Center, which is operated by the Smithsonian Astrophysical Observatory for NASA under contract PF2-130096. This work was supported in part by NSF grant PHY-1114374 and AST-0907890, as well as NASA grants NNX08AL43G and NNA09DB30A and NNX11AI49G. B.K. acknowledges support from NASA through Einstein Postdoctoral Fellowship Award No. PF9-00063 issued by the Chandra X-Ray Observatory Center, which is operated by the Smithsonian Astrophysical Observatory for and on behalf of the National Aeronautics Space Administration under contract NAS8-03060. N.Y. also thanks the Institute for Theory and Computation at the Harvard Smithsonian Center for Astrophysics for their hospitality.

\section{APPENDIX A}

\section{TWO-BODY SYSTEMS—EFFECTIVE ONE BODY}

GR pericenter precession has been studied in great detail and used to test Einstein's theory in the solar system, for example with observations of the perihelion precession of Mercury (e.g., Shapiro et al. 1972). The simplest method to derive such precession is to consider test-particle motion in an effective potential, assuming that GR introduces small corrections to Newtonian dynamics and small eccentricities (e.g., Misner et al. 1973, chap. 25, p. 668-670). ${ }^{13}$ The Hamiltonian (e.g., Artemova et al. 1996; Miller \& Hamilton 2002 ) is simply derived by integrating over the precession rate. Although this Hamiltonian leads to the correct ISCO location, if one uses the full expansion given in Artemova et al. (1996, Equation (4)), it is not equal to the 1PN Hamiltonian.

The purely orbital (non-spinning) 3PN Hamiltonian was derived in Jaranowski \& Schäfer (1998, 2001) (in the center of mass frame, and after subtraction of the total rest-mass term). Here, we focus only on expansions to 1PN order for a two-body system (with masses $m_{1}$ and $m_{2}$ and momenta $p_{1}$ and $p_{2}$, respectively). The Hamiltonian is then (e.g., Buonanno et al. 2006)

$$
\mathcal{H}_{1 P N}^{2 \text { body }}=-\frac{\left(m_{1}^{3}+m_{2}^{3}\right) p^{4}}{8 c^{2} m_{1}^{3} m_{2}^{3}}-\frac{k^{2}\left(3 m_{1}^{2}+7 m_{1} m_{2}+3 m_{2}^{2}\right) p^{2}}{2 c^{2} m_{1} m_{2} r}-\frac{k^{2}(\mathbf{p} \cdot \mathbf{r})^{2}}{2 c^{2} r^{3}}+\frac{k^{4}\left(m_{1}+m_{2}\right)^{2} \mu}{2 c^{2} r^{2}},
$$

where $\mathbf{r}$ is the radius vector between the two bodies, with magnitude $r$, the linear momentum of the effective one body problem is simply $p=-p_{1}=p_{2}$ and $\mu=m_{1} m_{2} /\left(m_{1}+m_{2}\right)$. Eliminating the short-period terms in the Hamiltonian, using the Von Zeipel transformation (for more details, see Brouwer 1959) for an orbit with SMA $a$ and eccentricity $e$, the double average Hamiltonian is

$\overline{13}$ Note that the same precession rate can be also derived directly from the 1PN metric (e.g., Misner et al. 1973, chap. 40, p. 1100-1112). 
given by

$$
\overline{\mathcal{H}}_{1 \mathrm{PN}}^{2 \mathrm{body}}=\frac{k^{4} \mu_{\mathrm{in}}\left(15 m_{1}^{2}+29 m_{1} m_{2}+15 m_{2}^{2}\right)}{8 a^{2} c^{2}}-\frac{3 k^{4} m_{1} m_{2}\left(m_{1}+m_{2}\right)}{a^{2} c^{2} \sqrt{1-e^{2}}},
$$

which is the same as Equation (28).

\section{APPENDIX B}

\section{THE VON ZEIPEL TRANSFORMATION}

The technique, known as the Von Zeipel transformation (for more details, see Brouwer 1959) is being used in order to eliminate the short-period terms in the Hamiltonian that depend of $l_{1}$ and $l_{2}$. The technique had been used to derive the double average hierarchical three-body Hamiltonian (e.g., Kozai 1962; Harrington 1968, 1969; Krymolowski \& Mazeh 1999; Naoz et al. 2013). Here the Hamiltonian we consider is simply $\mathcal{H}_{\text {tot }, 1 \mathrm{PN}}=\mathcal{H}_{\mathrm{N}}+\mathcal{H}_{1 \mathrm{PN}}$ (see Section 2). Following Naoz et al. (2013) Appendix A, we replace

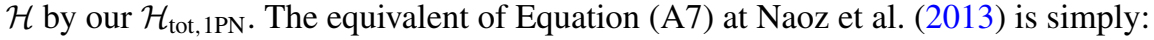

$$
\mathcal{H}_{\text {tot }, 1 \mathrm{PN}}=\mathcal{H}_{1}^{K}+\mathcal{H}_{2}^{K}+\mathcal{H}_{2}^{\mathrm{N}}+\mathcal{H}_{1 \mathrm{PN}},
$$

where $\mathcal{H}_{1}^{K}$ and $\mathcal{H}_{2}^{K}$ are the Kepler Hamiltonians that describe the inner and outer Newtonian orbits in the triple system, $\mathcal{H}_{2}^{\mathrm{N}}$ describes the Newtonian quadrupole interaction between the orbits (for the octupole interaction one can simply a $\mathcal{H}_{3}^{\mathrm{N}}$ ), and $\mathcal{H}_{1 \mathrm{PN}}$ describes the 1PN correction up to $\mathcal{O}\left(\alpha^{3}\right)$. In this technique, we use a canonical transformation that can eliminate the $l_{1}$ and $l_{2}$ terms from the $\mathcal{H}_{2}^{\mathrm{N}}+\mathcal{H}_{1 \mathrm{PN}}$ parts (which depends on $l_{1}$ and $l_{2}$ ), where the momenta are $p_{i} \in\left\{L_{i}, G_{i}, H_{i}\right\}$, and the coordinates are $q_{i} \in\left\{l_{i}, g_{i}, h_{i}\right\}$. Replacing $\mathcal{H}_{2}$ from Naoz et al. (2013) Appendix A, with $\mathcal{H}_{2}^{\text {tot }}=\mathcal{H}_{2}^{\mathrm{N}}+\mathcal{H}_{1 \mathrm{PN}}$, we find (after following their derivation) the equivalent of their Equation (A22):

$$
\mathcal{H}_{2}^{\mathrm{tot}, *}\left(q_{i}^{*}, p_{i}^{*}\right)=\frac{1}{4 \pi^{2}} \int_{0}^{2 \pi} d l_{1}^{*} d l_{2}^{*} \mathcal{H}_{2}^{\mathrm{tot}}\left(q_{i}^{*}, p_{i}^{*}\right),
$$

where the new momenta and coordinates have a superscript asterisk. Since Hamiltonian is an additive quantity, and the integral is an additive operation, the overall new Hamiltonian after the canonical transformation is simply

$$
\mathcal{H}_{2}^{\text {tot, } *}=\frac{1}{4 \pi^{2}}\left(\int_{0}^{2 \pi} d l_{1}^{*} d l_{2}^{*} \mathcal{H}_{2}^{\mathrm{N}}+\int_{0}^{2 \pi} d l_{1}^{*} d l_{2}^{*} \mathcal{H}^{1 \mathrm{PN}}\right) .
$$

Therefore we can simply use the double averaged Newtonian Hamiltonian derived in Naoz et al. (2013) and separately derive the double averaged 1PN Hamiltonian.

\section{APPENDIX C}

\section{EQUATION OF MOTIONS FOR THE 1PN INTERACTION}

Using the canonical relations (Equation (15)), we find the equations of motion for the interaction part of the 1PN Hamiltonian:

$$
\begin{aligned}
\left.\frac{d g_{1}}{d t}\right|_{1 \mathrm{PN}(\mathrm{int})}= & \frac{k^{4} m_{1} m_{2} m_{3}}{16 a_{2}^{3} c^{2}\left(1-e_{2}^{2}\right)^{3 / 2}\left(m_{1}+m_{2}\right)^{2}\left(m_{1}+m_{2}+m_{3}\right)}\left\{\frac { a _ { 1 } } { G _ { 1 } } ( m _ { 1 } + m _ { 2 } + m _ { 3 } ) \left[\left(1-e_{1}^{2}\right)\left(5 m_{1}^{2}-3 m_{1} m_{2}+5 m_{2}^{2}\right)\right.\right. \\
& \left.\left.-9 f_{m_{1} m_{2}}\left(\left(1-e_{1}^{2}\right) \cos 2 g_{1}+2 \cos ^{2} i_{\text {tot }} \sin ^{2} g_{1}\right)\right]+\frac{1}{G_{2}}\left[-8 f_{L L}+f_{i}\right]\right\}
\end{aligned}
$$

where $\tilde{L}_{1,2}=L_{1,2} / \mu_{\text {in,out }}$,

$$
\begin{gathered}
f_{m_{1} m_{2}}=m_{1}^{2}+m_{1} m_{2}+m_{2}^{2}, \\
f_{L L}=\tilde{L}_{1} \tilde{L}_{2}\left(m_{1}+m_{2}\right)\left(4\left(m_{1}+m_{2}\right)+3 m_{3}\right), \\
f_{e_{1}}=\left(2-5 e_{1}^{2}\right) m_{1}^{2}+3\left(-2+e_{1}^{2}\right) m_{1} m_{2}+\left(2-5 e_{1}^{2}\right) m_{2}^{2},
\end{gathered}
$$

and also

$$
f_{i}=3 a_{1}\left(m_{1}+m_{2}+m_{3}\right) \cos i_{\text {tot }}\left(f_{e_{1}}+3 e_{1}^{2} f_{m_{1} m_{2}} \cos 2 g_{1}\right)
$$

$$
\begin{aligned}
\frac{d g_{2}}{d t}= & -\frac{k^{4} m_{1} m_{2} m_{3}}{16 a_{2}^{3} c^{2}\left(1-e_{2}^{2}\right)^{3 / 2}\left(m_{1}+m_{2}\right)^{2}\left(m_{1}+m_{2}+m_{3}\right)} \\
& \times\left\{\frac{1}{G_{1}}\left[8 f_{L L}-f_{i}\right]-\frac{1}{2 G_{2}}\left[2 \cos i_{\text {tot }}\left(-8 f_{L L}+f_{i}\right)-16\left(m_{1}+m_{2}\right)^{2} \cos i_{\text {tot }} \tilde{L}_{1} \tilde{L}_{2}\left[16\left(m_{1}+m_{2}\right) \tilde{L}_{1} \tilde{L}_{2}\left(7\left(m_{1}+m_{2}\right)+6 m_{3}\right) \cos i_{\text {tot }}\right.\right.\right. \\
& \left.\left.+\frac{3}{2} a_{1}\left(m_{1}+m_{2}+m_{3}\right)\left(-f_{e_{1}}\left[1+3 \cos 2 i_{\mathrm{tot}}\right]+18 e_{1}^{2} f_{m_{1} m_{2}} \cos 2 g_{1} \sin ^{2} i_{\mathrm{tot}}\right)\right]\right\}
\end{aligned}
$$




$$
\left.\frac{d e_{1}}{d t}\right|_{1 \mathrm{PN}(\mathrm{int})}=\frac{9 a_{1} e_{1} \sqrt{1-e_{1}^{2}} k^{4} m_{1} m_{2}\left(m_{1}^{2}+m_{1} m_{2}+m_{2}^{2}\right) m_{3} \sin ^{2} i_{\mathrm{tot}} \sin \left(2 g_{1}\right)}{16 a_{2}^{3} c^{2}\left(1-e_{2}^{2}\right)^{3 / 2} L_{1}\left(m_{1}+m_{2}\right)^{2}} .
$$

The change of the inner orbital angular momentum is simply

$$
\left.\frac{d G_{1}}{d t}\right|_{1 \mathrm{PN}(\mathrm{int})}=\frac{9 a_{1} e_{1}^{2} k^{4} m_{1} m_{2}\left(m_{1}^{2}+m_{1} m_{2}+m_{2}^{2}\right) m_{3} \sin ^{2} i_{\mathrm{tot}} \sin \left(2 g_{1}\right)}{16 a_{2}^{3} c^{2}\left(1-e_{2}^{2}\right)^{3 / 2}\left(m_{1}+m_{2}\right)^{2}},
$$

while for the outer orbit it is simply zero. Thus,

$$
\left.\frac{d H_{1}}{d t}\right|_{1 \mathrm{PN}(\mathrm{int})}=\frac{\sin i_{2}}{\sin i_{\mathrm{tot}}} \frac{d G_{1}}{d t}
$$

i.e.,

$$
\left.\frac{d H_{1}}{d t}\right|_{1 \mathrm{PN}(\mathrm{int})}=\frac{\sin i_{2}}{\sin i_{\mathrm{tot}}} \frac{9 a_{1} e_{1}^{2} k^{4} m_{1} m_{2}\left(m_{1}^{2}+m_{1} m_{2}+m_{2}^{2}\right) m_{3} \sin ^{2} i_{\mathrm{tot}} \sin \left(2 g_{1}\right)}{16 a_{2}^{3} c^{2}\left(1-e_{2}^{2}\right)^{3 / 2}\left(m_{1}+m_{2}\right)^{2}} .
$$

The inclinations evolve according to $\left(\cos i_{1}\right)=\dot{H}_{1} / G_{1}-\dot{G}_{1} / G_{1} \cos i_{1}$ (e.g., Naoz et al. 2013), and thus,

$$
\left(\left.\dot{\left.\cos i_{1}\right)}\right|_{1 \mathrm{PN}(\mathrm{int})}=\frac{9 a_{1} e_{1}^{2} k^{4} m_{1} m_{2}\left(m_{1}^{2}+m_{1} m_{2}+m_{2}^{2}\right) m_{3} \sin ^{2} i_{\mathrm{tot}} \sin \left(2 g_{1}\right)}{16 a_{2}^{3} c^{2}\left(1-e_{2}^{2}\right)^{3 / 2}\left(m_{1}+m_{2}\right)^{2}} \frac{1}{G_{1}}\left(\frac{\sin i_{2}}{\sin i_{\mathrm{tot}}}-\cos i_{1}\right),\right.
$$

and since $\left(\cos i_{2}\right)=\dot{H}_{2} / G_{2}-\dot{G}_{2} / G_{2} \cos i_{2}$ and $\dot{H}_{2}=-\dot{H}_{1}$ (e.g., Naoz et al. 2013) we find

$$
\left(\cos i_{2}\right)=\frac{\sin i_{2}}{G_{2} \sin i_{\mathrm{tot}}} \frac{9 a_{1} e_{1}^{2} k^{4} m_{1} m_{2}\left(m_{1}^{2}+m_{1} m_{2}+m_{2}^{2}\right) m_{3} \sin ^{2} i_{\mathrm{tot}} \sin \left(2 g_{1}\right)}{16 a_{2}^{3} c^{2}\left(1-e_{2}^{2}\right)^{3 / 2}\left(m_{1}+m_{2}\right)^{2}} .
$$

\section{REFERENCES}

Amaro-Seoane, P., Aoudia, S., Babak, S., et al. 2012, CQGra, 29, 124016

Amaro-Seoane, P., Aoudia, S., Babak, S., et al. 2013, GWN, 6, 4

Amaro-Seoane, P., Sesana, A., Hoffman, L., et al. 2010, MNRAS, 402, 2308

Antonini, F., \& Perets, H. 2012, ApJ, 757, 27

Armitage, P. J., \& Natarajan, P. 2005, ApJ, 634, 921

Artemova, I. V., Bjoernsson, G., \& Novikov, I. D. 1996, ApJ, 461, 565

Arun, K., Iyer, B. R., Sathyaprakash, B., \& Sinha, S. 2007a, PhRvD, 75,124002

Arun, K., Iyer, B. R., Sathyaprakash, B., Sinha, S., \& Broeck, C. V. D. 2007b, PhRvD, 76, 104016

Arun, K. G., Blanchet, L., Iyer, B. R., \& Sinha, S. 2009, PhRvD, 80, 124018

Blaes, O., Lee, M. H., \& Socrates, A. 2002, ApJ, 578, 775

Brouwer, D. 1959, AJ, 64, 378

Brown, D. A., \& Zimmerman, P. J. 2010, PhRvD, 81, 024007

Buonanno, A., Chen, Y., \& Damour, T. 2006, PhRvD, 74, 104005

Damour, T., \& Deruelle, N. 1985, JAF, 25, 21

Dirac, P. A. M. 1950, Can. J. Math., 2, 937

Dotti, M., Sesana, A., \& Decarli, R. 2012, AdAst, 2012, 940568

Eggleton, P. P., Kisseleva-Eggleton, L., \& Dearborn, X. 2007, in IAU Symp.

240, Binary Stars as Critical Tools \& Tests in Contemporary Astrophysics,

ed. W. I. Hartkopf, E. F. Guinan, \& P. Harmanec (Cambridge: Cambridge

Univ. Press), 347

Fabrycky, D., \& Tremaine, S. 2007, ApJ, 669, 1298

Finn, L. S., \& Lommen, A. N. 2010, ApJ, 718, 1400

Ford, E. B., Joshi, K. J., Rasio, F. A., \& Zbarsky, B. 2000a, ApJ, 528, 336

Ford, E. B., Kozinsky, B., \& Rasio, F. A. 2000b, ApJ, 535, 385

Galaviz, P., \& Brügmann, B. 2011, PhRvD, 83, 084013

Harrington, R. S. 1968, AJ, 73, 190

Harrington, R. S. 1969, CeMec, 1, 200

Hoffman, L., \& Loeb, A. 2007, MNRAS, 377, 957

Holman, M., Touma, J., \& Tremaine, S. 1997, Natur, 386, 254

Innanen, K. A. 1979, AJ, 84, 960

Innanen, K. A. 1980, AJ, 85, 81

Ivanova, N., Chaichenets, S., Fregeau, J., et al. 2010, ApJ, 717, 948

Jaranowski, P., \& Schäfer, G. 1998, PhRvD, 57, 7274

Jaranowski, P., \& Schäfer, G. 2001, PhRvD, 63, 029902

Katz, B., Dong, S., \& Malhotra, R. 2011, PhRvL, 107, 181101
Kiseleva, L. G., Eggleton, P. P., \& Mikkola, S. 1998, MNRAS, 300, 292

Kocsis, B., \& Levin, J. 2012, PhRvD, 85, 123005

Kocsis, B., Ray, A., \& Portegies Zwart, S. 2012, ApJ, 752, 67

Kozai, Y. 1962, AJ, 67, 591

Krymolowski, Y., \& Mazeh, T. 1999, MNRAS, 304, 720

Kulkarni, G., \& Loeb, A. 2012, MNRAS, 422, 1306

Lidov, M. L. 1962, P\&SS, 9, 719

Lidov, M. L., \& Ziglin, S. L. 1976, CeMec, 13, 471

Lithwick, Y., \& Naoz, S. 2011, ApJ, 742, 94

Lousto, C. O., \& Nakano, H. 2008, CQGra, 25, 195019

Mandel, I., Brown, D. A., Gair, J. R., \& Miller, M. C. 2008, ApJ, 681, 1431

Marchal, C. (ed.) 1990, The Three-body Problem (Amsterdam: Elsevier)

Mardling, R. A. 2007, MNRAS, 382, 1768

Mardling, R. A., \& Aarseth, S. J. 2001, MNRAS, 321, 398

Mazeh, T., \& Shaham, J. 1979, A\&A, 77, 145

McKenna, J., \& Lyne, A. G. 1988, Natur, 336, 226

Miller, M. C., \& Hamilton, D. P. 2002, ApJ, 576, 894

Misner, C. W., Thorne, K. S., \& Wheeler, J. A. (ed.) 1973, Gravitation (San Francisco, CA: Freeman)

Moore, C. 1993, PhRvL, 70, 3675

Morais, M. H. M., \& Giuppone, C. A. 2012, MNRAS, 424, 52

Murray, C. D., \& Dermott, S. F. (ed.) 2000, Solar System Dynamics (Princeton, NJ: Princeton Univ. Press)

Naoz, S., Farr, W. M., Lithwick, Y., Rasio, F. A., \& Teyssandier, J. 2011, Natur, 473,187

Naoz, S., Farr, W. M., Lithwick, Y., Rasio, F. A., \& Teyssandier, J. 2013, MNRAS, 431, 2155

Naoz, S., Farr, W. M., \& Rasio, F. A. 2012, ApJL, 754, L36

Nowak, M. A., \& Wagoner, R. V. 1991, ApJ, 378, 656

O'Leary, R. M., Kocsis, B., \& Loeb, A. 2009, MNRAS, 395, 2127

O’Leary, R. M., Rasio, F. A., Fregeau, J. M., Ivanova, N., \& O'Shaughnessy, R. 2006, ApJ, 637, 937

Perets, H. B., \& Fabrycky, D. C. 2009, ApJ, 697, 1048

Peters, P. C. 1964, PhRv, 136, 1224

Pribulla, T., \& Rucinski, S. M. 2006, AJ, 131, 2986

Prodan, S., \& Murray, N. 2012, ApJ, 747, 4

Schäfer, G. 1987, PhLA, 123, 336

Sesana, A. 2010, ApJ, 719, 851

Seto, N. 2012, PhRvD, 85, 064037 
Shapiro, I. I., Pettengill, G. H., Ash, M. E., et al. 1972, PhRvL, 28, 1594

Sharpee, B. J., \& Thompson, T. A. 2013, ApJ, 766, 64

Takeda, G., Kita, R., \& Rasio, F. A. 2008, ApJ, 683, 1063

Thompson, T. A. 2011, ApJ, 741, 82

Tokovinin, A. A. 1997, AstL, 23, 727

Valtonen, M., \& Karttunen, H. (ed.) 2006, The Three-Body Problem (Cambridge: Cambridge Univ. Press)
Valtonen, M. J. 1996, MNRAS, 278, 186

Wen, L. 2003, ApJ, 598, 419

Wu, Y., Murray, N. W., \& Ramsahai, J. M. 2007, ApJ, 670, 820

Yunes, N., Arun, K., Berti, E., \& Will, C. M. 2009, PhRvD, 80, 084001

Yunes, N., Miller, M. C., \& Thornburg, J. 2011, PhRvD, 83, 044030

Zhang, K., Hamilton, D. P., \& Matsumura, S. 2013, arXiv:1302.1620 